\title{
Model-based wavefront control for CCAT
}

David Redding, John Z. Lou, Andy Kissil, Matt Bradford, Steve Padin, et al.

David Redding, John Z. Lou, Andy Kissil, Matt Bradford, Steve Padin, David Woody, "Model-based wavefront control for CCAT," Proc. SPIE 8336, Integrated Modeling of Complex Optomechanical Systems, 83360Q (10 November 2011); doi: 10.1117/12.916694

SDIE Event: Integrated Modeling of Complex Optomechanical Systems, 2011, Kiruna, Sweden 


\title{
Model-Based Wavefront Control for CCAT
}

\author{
David Redding ${ }^{\mathrm{a}}$, John Z. Lou ${ }^{\mathrm{a}}$, Andy Kissil ${ }^{\mathrm{a}}$, Matt Bradford ${ }^{\mathrm{a}}$, Steve Padin ${ }^{\mathrm{b}}$ and David Woody \\ a Jet Propulsion Laboratory, California Institute of Technology, Pasadena CA 91109 \\ ${ }^{\mathrm{b}}$ California Institute of Technology, Pasadena CA 91106
}

\begin{abstract}
The $25-\mathrm{m}$ aperture CCAT submillimeter-wave telescope will have a primary mirror that is divided into 162 individual segments, each of which is provided with 3 positioning actuators. CCAT will be equipped with innovative Imaging Displacement Sensors (IDS) - inexpensive optical edge sensors - capable of accurately measuring all segment relative motions. These measurements are used in a Kalman-filter-based Optical State Estimator to estimate wavefront errors, permitting use of a minimum-wavefront controller without direct wavefront measurement. This controller corrects the optical impact of errors in 6 degrees of freedom per segment, including lateral translations of the segments, using only the 3 actuated degrees of freedom per segment. The edge sensors do not measure the global motions of the Primary and Secondary Mirrors. These are controlled using a gravity-sag look-up table. Predicted performance is illustrated by simulated response to errors such as gravity sag.
\end{abstract}

Keywords: Segmented mirror, wavefront control, edge sensors, Kalman filter.

\section{Nomenclature}

$\mathrm{x}_{\mathrm{R}}$, rigid-body state of each optic or optical assembly, $\left[\mathrm{x}_{\mathrm{R}}\right]=(6 *$ nopt, 1$)$

$\mathrm{x}_{\mathrm{D}}$, segment deformation state, $\left[\mathrm{x}_{\mathrm{D}}\right]=\left(\right.$ ndef $^{*} n$ seg, 1$)$

$\mathrm{u}_{\mathrm{R}}$, segment rigid body control command, $\left[\mathrm{u}_{\mathrm{R}}\right]=\left(6^{*} n\right.$ seg, 1$)$

$\mathrm{u}_{\mathrm{SM}}, \mathrm{SM}$ rigid body control command, $\left[\mathrm{u}_{\mathrm{SM}}\right]=(5,1)$

$\mathrm{u}_{\mathrm{c}}$, pointing control command, $\left[\mathrm{u}_{\mathrm{c}}\right]=(2,1)$

$\delta \mathrm{T}$, temperature change, $[\delta \mathrm{T}]=\left(n T^{*} n\right.$ seg, 1$)$

$\mathrm{x}$, process noise, $[\mathrm{dT}]=(\mathrm{x}, 1)$

1, optical edge sensor measurements, $[1]=(2 * n l, 1)$

$\delta 1$, optical edge measurement noise, $[\delta 1]=(2 * n l, 1)$

$\mathrm{w}$, wavefront, $[\mathrm{w}]=($ nray, 1$)$

$\mathrm{w}_{\mathrm{Atm}}$, wavefront error due to atmosphere

$\delta \mathrm{w}$, wavefront measurement noise, $[\delta \mathrm{w}]=($ nray, 1$)$

OPD, Optical Path Difference matrix

c, ray centroid, $[\mathrm{c}]=(2,1)$

$\delta \mathrm{c}$, pointing measurement noise, $[\delta \mathrm{c}]=(2,1)$

$\mathrm{K}$, estimator gain

$\mathrm{c}_{\mathrm{wu}}$ control weighting coefficient

i, time index

Capitalized variable signifies the covariance of that variable

Bar over a variable signifies a predicted value (prior to incorporation of current measurement data)

Hat over a variable signifies current estimate

Squiggle over a variable signifies current estimate error

Integrated Modeling of Complex Optomechanical Systems, edited by Torben Andersen, Anita Enmark, Proc. of SPIE Vol. 8336, 83360Q - @ 2011 SPIE - CCC code: 0277-786X/11/\$18 - doi: 10.1117/12.916694 


\section{Introduction}

CCAT will be a $25-\mathrm{m}$ aperture submillimeter-wave telescope, operating at wavelengths between $200 \mathrm{um}$ and $2.5 \mathrm{~mm}$. It will be built at an altitude of 5,612 m (18,410 ft), near Cerro Chajnantor in the Atacama desert of northern Chile [1]. The CCAT Primary Mirror (PM) will be assembled from 162 "keystone" segments, which must be precisely co-aligned and co-phased with each other, so that the primary mirror surface is smooth to a small fraction of the shortest wavelength: to about 20 um RMS Wavefront Error (WFE). The PM as a whole must also remain in good alignment with the Secondary Mirror (SM) and cameras.

This paper presents a design for the CCAT Wavefront Control (WFC) system, which will keep the 162 PM segments, and the SM, in good alignment during science operations. The WFC system will use 3 positioning actuators on each segment, and 6 actuators on the SM, to move each optic as needed to reject disturbances. Optical edge sensors will continuously measure segment-to-segment alignments; real-time estimation and control calculations will determine actuator commands that keep all PM segments phased and Wavefront Errors (WFE) small. Initial alignment and periodic recalibration of the optics will be performed using direct Wavefront Sensing (WFS) techniques. Control of the global SM and PM tilt, decenter and focus will be performed using precalibrated offsets stored in a look-up table - direct measurements of $\mathrm{PM} / \mathrm{SM}$ collimation or despace are not currently planned. A more detailed report on the WF control system is provided in [2].

The CCAT WFC system is being designed and evaluated using an integrated model of the telescope: a computer simulation of the structure and optics, including sensors and controls, that can be used to predict response to disturbances such as gravity sag, temperature changes, or fabrication and assembly errors. The integrated model, briefly described in this paper, is used in parametric studies to determine component specifications, such as sensor noise, actuator noise, structural alignment precision, and thermal sensitivities, for consistency with the CCAT WFE budget.

Parts of the model are also used directly in the estimation and control calculations, for prediction of gravity sag, for instance, in the determination of the gravity look-up table used to compensate global SM and PM motions as the telescope elevation angle changes. Linearized models are directly embedded into the WF estimation and control calculations, so that edge sensor measurements can be projected to WF space, to estimate WFE from indirect, structural measurements. This allows use of a minimum-WFE control law without directly sensing the WF, which is essential for accurately compensating the optical effects of displacements in uncontrolled degrees-of-freedom (DOF), such as lateral motions of the PM segments.

\section{CCAT Overview}

Optics. The CCAT telescope is a Ritchey-Chretien design with a 25 -meter aperture, a primary mirror focal ratio of 0.4 , and a system focal ratio of 6.0, as described in Refs. [1], [3] and [6]. CCAT optical performance is modeled using the MACOS optical modeling code [4], which combines ray trace and Fourier physical optics as needed to predict the performance of the telescope. MACOS is typically called directly from MATLAB ${ }^{\mathrm{TM}}$, which is used as a simulation environment to facilitate integration of optics and structures models.

A ray trace of the design, and the nominal aperture Optical Path Difference (OPD, or WFE) plot, are shown in Figure 1. The current design uses 162 separate Primary Mirror (PM) segments, each about $2 \mathrm{~m}$ across. Light entering the telescope hits 3 mirrors: the segmented PM; then the Secondary Mirror (SM), which is about $9 \mathrm{~m}$ above the PM, and then a Fold Mirror (FM), located $3 \mathrm{~m}$ behind the PM. Ultimately the light will be directed to multiple imaging and spectroscopic instruments, but for the purposes of this report, the telescope focal plane will be as shown, just after the FM, behind the PM. The telescope exit pupil is located at a virtual image of the primary, approximately $12 \mathrm{~m}$ behind the FM - a reference surface located at this pupil is used to evaluate OPD or WFE plots.

Perturbations of the optics, due to manufacturing or assembly errors, thermal expansion, gravity sag, actuator motions, or arbitrary user inputs, cause deviations from the perfect, as-designed optical prescription. Rigid-body perturbations of each optic are due to deformations of the underlying support structures, and are implemented in the optics model by rotations and translations in local coordinates, centered on the middle of the facesheet of each optic. Surface deformations of each optic are represented by a matrix or grid of surface points, with each element of the matrix representing a surface height relative to the base conic surface. Surface deformation maps are added to the base conic surface in the MACOS prescription, to define the deformed surface for propagating light through the system. 
Structures. The current CCAT Finite Element Model (FEM) structural model, shown in Figure 2, is based on a preliminary design developed by D. Woody, et al [5], and is composed of a steel axle, a CFRP primary and secondary support truss, and actuated CFRP primary segment rafts, each with a fixed array $(2 \times 2)$ of aluminum reflector panels. The support truss is composed of CFRP struts connected via Invar node-balls. There are 162 CFRP primary segment rafts which are each kinematically mounted to the support truss, and thermally insulated to provide a nearly isothermal, and very stable platform.

The CCAT FEM represents the support truss structure using "CROD" rod elements, with equivalent properties for the strut/node-ball assembly. The model has approximately $120 \mathrm{~K}$ nodes, and less than 1 million degrees of freedom: a manageable size with today's compute power, even without model reduction. As the design matures we will add more detail and transition to super-element modeling.

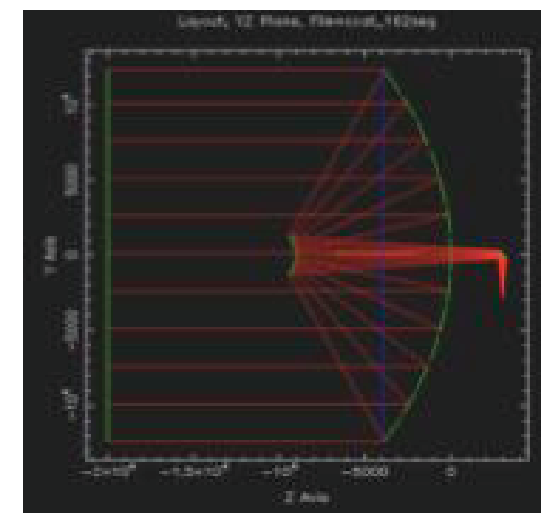

Main aperture $=25 \mathrm{~m}$

$\mathrm{PM} \mathrm{F/ \#} \mathrm{=0.4}$

System $\mathrm{F} / \#=6.0$

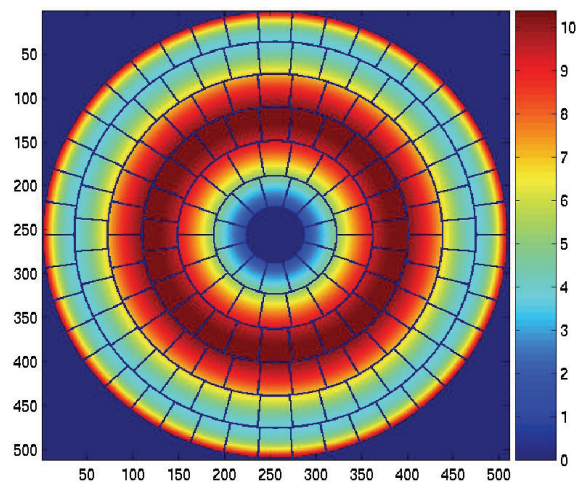

Figure 1. Left image: CCAT optical system layout. Right image: OPD or WFE plot showing 6-ring segmentation with 162 keystone elements.

Figure 2 also shows one of the segment raft assembly models: a ring 3 segment in this case. Each raft is flexure-mounted to give a kinematic interface to the truss, and has three actuators, controlling piston, tip and tilt motion of the raft. The raft sub-base, shown in light blue, is composed of CFRP face-sheets, top and bottom, modeled with plate elements, and an aluminum honeycomb core, modeled with equivalent solid elements having orthotropic material properties. The triangular frame, shown in orange, provides support for the truss-end of the three actuators, minimizing relative motion between the actuator bases. The green cylinders represent the 3 segment piston/tip/tilt actuators.

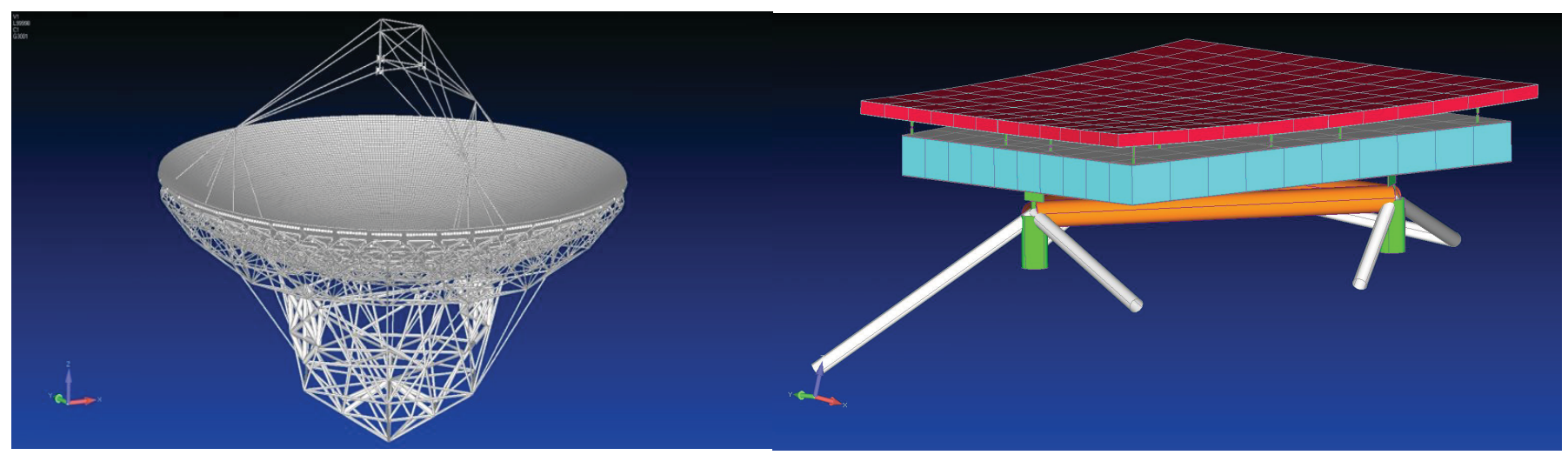

Figure 2. Left Image: CCAT Structural FEM. Right Image: Raft Assembly FEM for a Ring 3 Segment.

Nodes representing edge sensors were also attached to each raft. The sensor node attachments were implemented using simple rigid links to the nearest raft nodes. The response data passed to the controller subtracts the sensor displacements/rotations due to segment rigid body motion.

There are four aluminum panels ( $2 \times 2$ array) mounted to the CFRP sub-frame using five flexured stand-offs. The current panel design is an all-aluminum honeycomb sandwich structure. The invar stand-offs and raft-mount flexures are modeled using beam elements. 
The secondary mirror is modeled simply at this time, using lumped masses, and the secondary support struts are modeled using beam elements with CFRP material properties. This is sufficient to capture first-order effects for thermal, gravity and dynamic disturbances.

Gravity sag example. The telescope pointing elevation angle will change continuously during CCAT observations, to compensate for earth rotation, and to change observational targets. This causes the direction of gravity relative to the telescope structure to also change, which is the single largest disturbance expected during normal operations. These gravity changes have 2 main effects:

- The PM and SM substructures deform, leading to segment rigid-body errors, including relative and global modes.

- Much smaller changes occur in internal segment structure, changing segment figure and directly adding WFE; it also distorts the IDS mounting positions, causing the edge sensors to change orientation slightly, leading to a small spurious segment position estimation error.

Figure 3 shows gravity sag effects in a particular example case, where the telescope was aligned while in a zenithpointing attitude, and then tilted to $45^{\circ}$ from zenith. The PM segment and SM rigid body perturbations are shown in the left hand plot; the right hand plot shows the effect on segment alignments, with perturbations ranging from 1.4 to -2.3 $\mathrm{mm}$. Figure 4 shows the figure error induced on a typical segment, which is much smaller at $0.643 \mathrm{um}$ RMS across the reflecting surface. The figure error can be reduced further, to less than 0.4 microns, by optimizing the sub-frame support point locations. WFE performance in this example is discussed later, in Section 7.

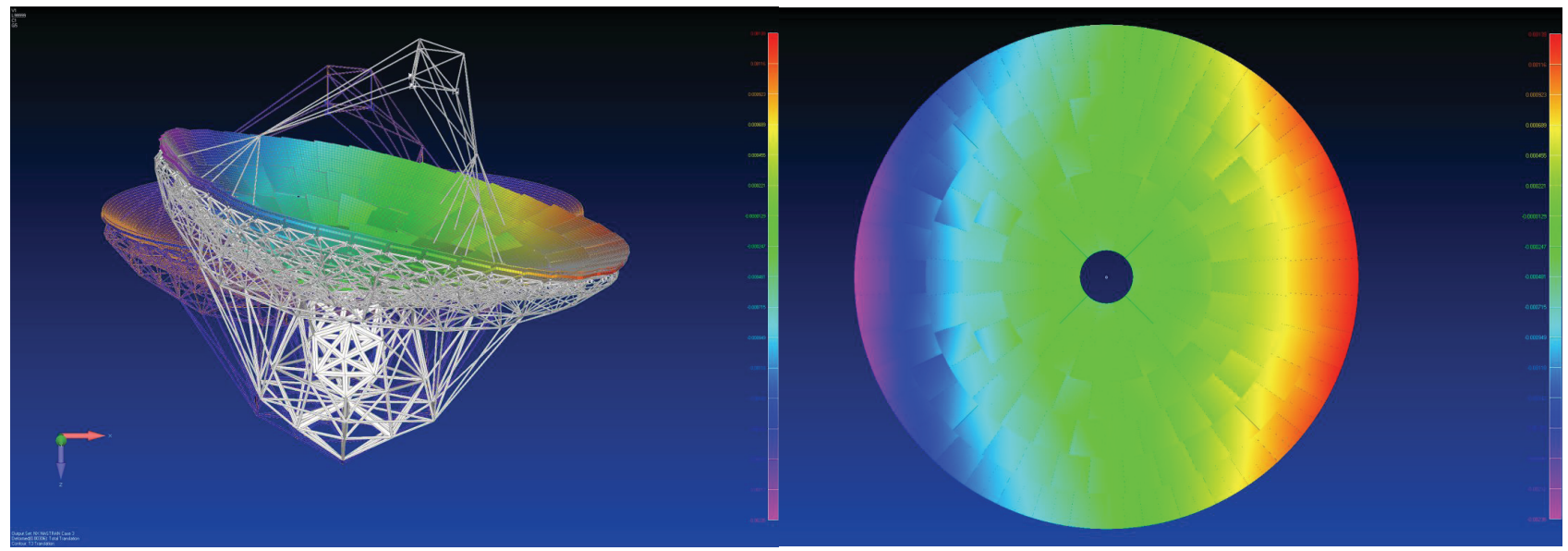

Figure 3. Structural model prediction of CCAT structural deformation incurred as the elevation axis is driven from zenith to $45^{\circ}$ elevation.

We have performed some dynamical analysis of the telescope, to verify integrity of the structural design and model. The telescope system normal modes have been computed: $1^{\text {st }}$ and $2^{\text {nd }}$ (rocking) mode frequencies are 7.5 and $9.0 \mathrm{~Hz}$, respectively. Evaluation of the telescope response to both internal and external sources of dynamic disturbances will be performed in future work. Sources for internally induced vibration are telescope nodding motions, used during observations to calibrate background noise; plus higher frequency effects such as drive motor torque ripple \& axis bearing friction. Sources for externally induced vibration are mainly due to wind: vortex shedding, wind gusting, and enclosure/foundation born vibration propagation to the telescope pier. Projections at this point in the design cycle are that dynamical disturbances do not require active compensation. Future work will simulate the closed-loop dynamical response of the telescope to representative dynamical disturbances in detail to verify this conclusion.

Optical Edge Sensors: the CCAT "Imaging Displacement Sensor." Edge sensors provide a means for measuring the displacement and rotation of one PM segment with respect to another, adjacent segment. By equipping all segment edges with edge sensors, a chain of measurements is created, which can be processed in a computer to estimate the total figure of the PM. Edge sensors have been successfully used for large telescopes in the past, notably the $10 \mathrm{~m}$ aperture, 36 segment Keck telescopes, which uses capacitive edge sensors; they are also planned for use by the Thirty Meter Telescope and other future telescopes. Electronic edge sensors using capacitive or inductive pickoff mechanisms are commercially available, but are too expensive for CCAT application. 


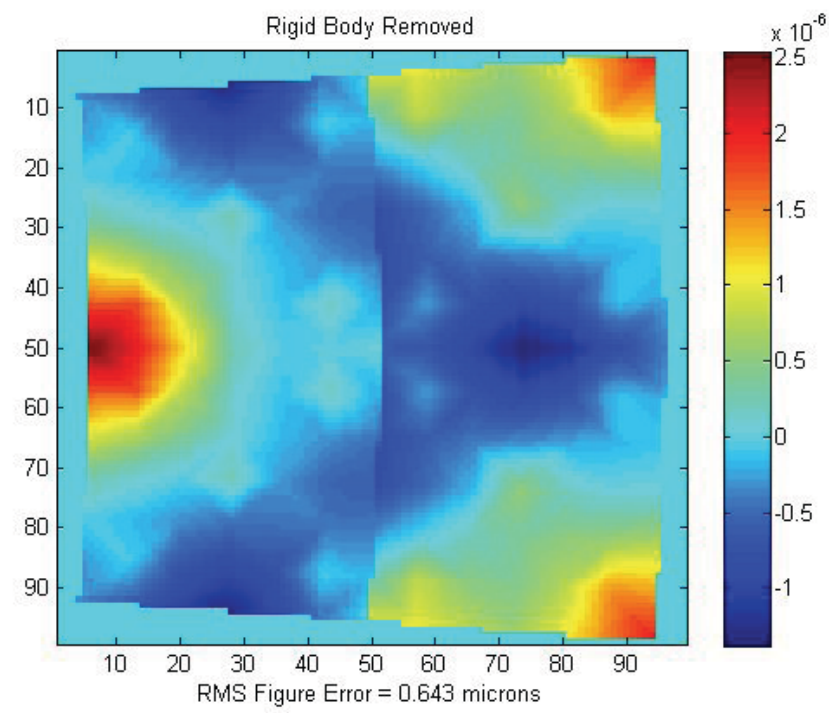

Figure 4. Ring 6 Segment Surface Figure Error due to $45^{\circ}$ elevation angle change gravity sag.

The CCAT Imaging Displacement Sensors (IDS) are optical edge sensors with nanometer-level accuracy [9]. They are highly linear, with consistent performance over a range of multiple millimeters. They are inexpensive, utilizing conventional commercial hardware. IDS properties include:

IDS measurements are linear, even with large deflections. This comes from using beams of light measured on commercial grade CCD or CMOS detectors, which avoids high-order electric field effects inherent to electronic edge sensors.

- When properly configured, 2 pairs of IDS sensor heads can measure all 6 DOF of relative motion between 2 adjacent segments with good accuracy.

- IDS sensor heads can be located close together or far apart. This provides design flexibility, for instance to reduce measurement error sensitivity due to segment thermal deformations.

Optical edge sensors were first proposed for CCAT by Alex Abramovici (JPL), who tested a device consisting of a collimated laser beam on one segment, and a quadrant detector on the adjacent segment, to sense lateral translation at a distance. A lab device [12] demonstrated high dihedral angle sensitivity and good stability and sensitivity for ranges on the order of $10 \mathrm{~mm}$, but was less accurate for distances of $\sim 100 \mathrm{~mm}$ or more.

The IDS takes a different approach, projecting a simple image defined by a light source and a shadow mask collimator on one sensor head, onto a bare camera CCD or CMOS chip on a second, remote sensor head. The light source for the collimated image is an inexpensive LED with a pinhole directly in front of the LED, and a shadow mask (nominally a circle) located $\sim 100 \mathrm{~mm}$ away. The resulting beam of light is projected across the gap between segments, to fall on the CCD or CMOS detector. The position of the image can be very precisely estimated in 2 axes, to provide measurements of relative segment motion. The size of the pinhole, shadow mask, distance to the CCD chip, and diffraction effects determine the size of the image on the chip. Figure 6 shows the sensor configuration.

IDS computer processing includes image preprocessing and centroiding functions, described in detail in [9]. The center finding software uses a Fourier Transform Symmetry algorithm (rather than a centroiding algorithm) to find the symmetry point of the image [9].

Experiments with a single prototype IDS sensor have shown excellent basic performance. The prototype sensor utilizes a Sony 640 x 480 format, 7.2 um pixel CCD detector run at $30 \mathrm{fps}$. Measurements taken regularly over 3 months by the prototype in a facility environmentally controlled to $\pm 3 \mathrm{C}$ shows single measurement performance better than $50 \mathrm{~nm}$; performance better than $10 \mathrm{~nm}$ for $30 \mathrm{~Hz}$ measurements averaged over $1 \mathrm{sec}$; and, averaging over intervals ranging from $50 \mathrm{sec}$ to $1000 \mathrm{sec}$, performance was better than $1 \mathrm{~nm}$ (Figure 5). Systematic drift or aging effects were less than 50 $\mathrm{nm} / \mathrm{month}$. Other results for the prototype IDS are presented in [9]. 


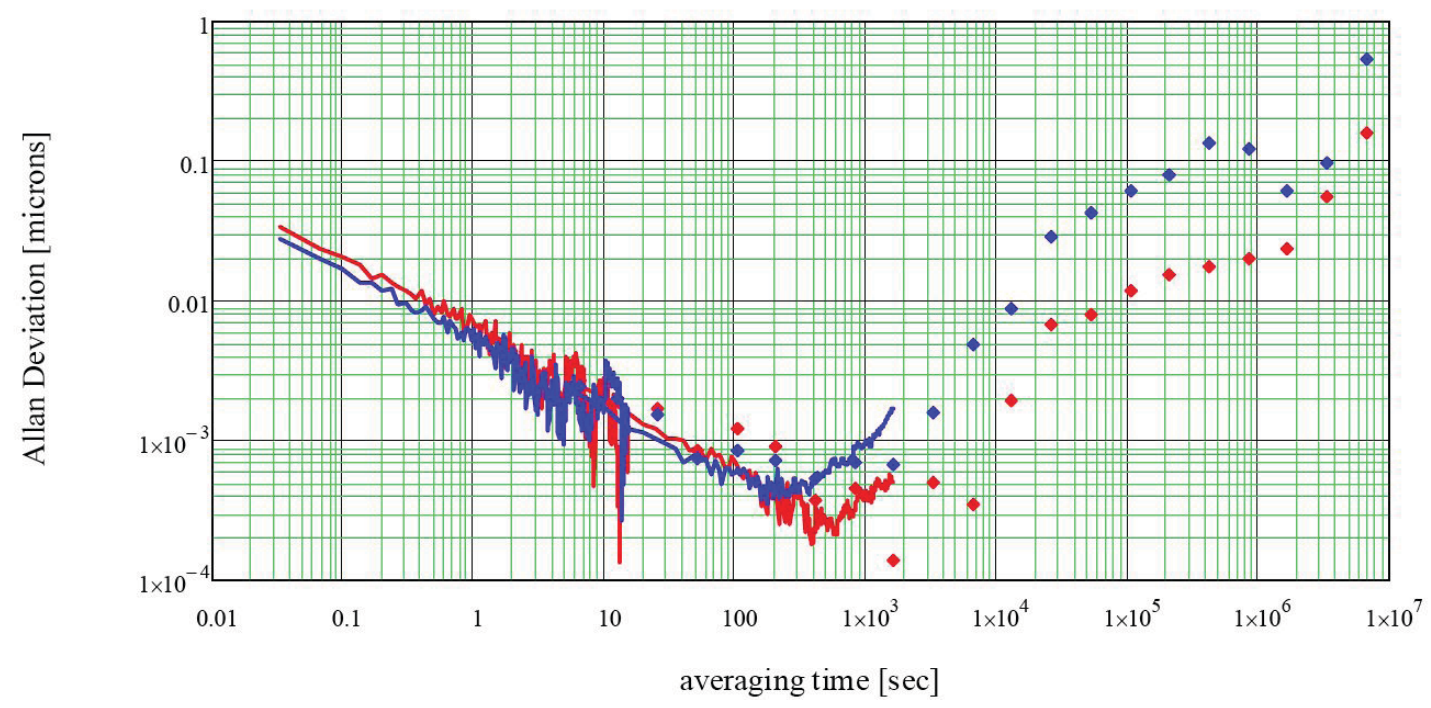

Figure 5. Allan Deviation, showing $\mathrm{x}$ (blue) and $\mathrm{y}$ (red) spot positions measured over a 4 month period. Accuracy for single measurements was $<100 \mathrm{~nm}$; for measurements averaged over $10 \mathrm{sec},<4 \mathrm{~nm}$.

The 2 ( $\mathrm{y}$ and $\mathrm{z}$ ) measurements made by a single IDS beam, with one collimator and one detector, cannot distinguish between piston $\left(\delta_{z}\right)$ and dihedral angle $\left(\theta_{y}\right)$ on the one hand, and lateral shear $\left(\delta_{y}\right)$ and segment twist $\left(\theta_{z}\right)$ on the other. These motions can be separated, however, if 2 IDS are used, with the collimator and CCD chip positions reversed and the 2 light beams parallel, as shown in Figure 6. A complete CCAT "IDS sensor pair" thus consists of 2 IDS sensor heads, each of which contains both a collimator and a detector.

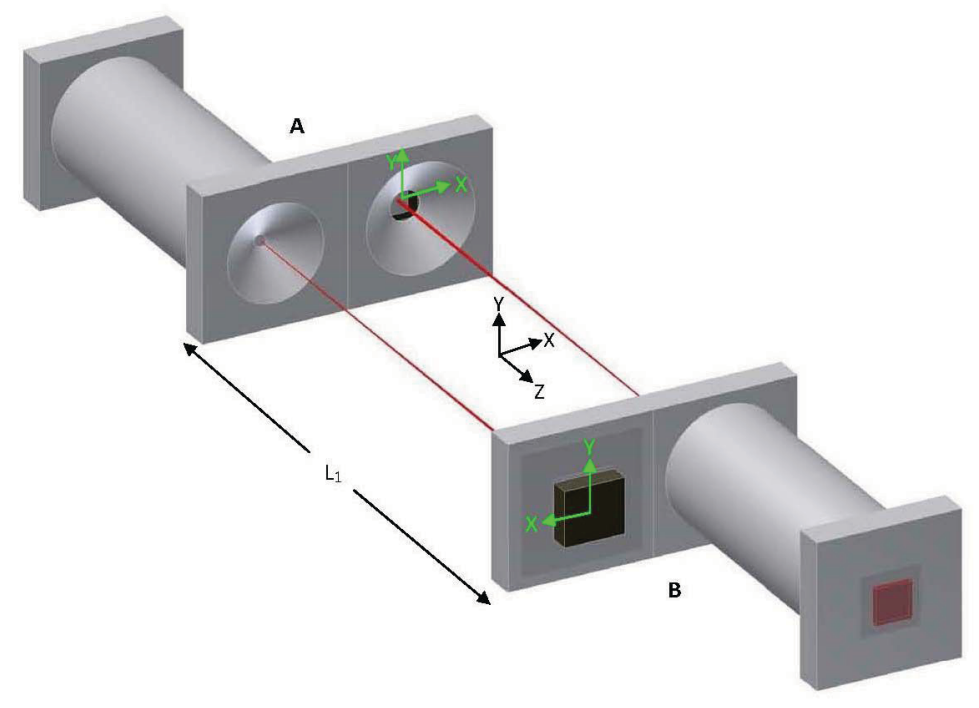

Figure 6. A single IDS (“Optical Edge Sensor," or OES) sensor pair, with 2 beams in parallel. Each IDS Sensor Head contains: a light source consisting of an LED and a shadow mask; and a CMOS or CCD detector.

CCAT segments will be equipped with 2 IDS sensor pairs per segment edge, as sketched in Figure 7. By separating the 2 pairs, a lever arm is created, so that rotation about the gap direction $\left(\theta_{\mathrm{x}}\right)$ can be seen. Also, by angling the sensor pairs at $+45^{\circ}$ and $-45^{\circ}$ to the edge, motions in the gap direction $\left(\delta_{\mathrm{x}}\right)$ can be seen. The configuration of Figure 7 allows 2 sensor pairs, with a total of 8 measurements (y and $\mathrm{z}$ on 4 detectors), to form a linear basis of rank 6 (with redundancy), that provides robust, unambiguous measurements of all 6 DOFs of relative segment motion, as illustrated in Figure 8. 
Using 2 IDS pairs per segment edge, one can extend a chain of measurements beyond a single pair of segments: Segment 2 is measured relative to segment 1; segment 3 is measured relative to segment 2; etc. Since there are generally multiple rings of segments in larger telescopes, each segment can be measured relative to every other segment via multiple different chains, providing a high level of redundancy, and averaging down the effect of measurement errors and noise. The result is robust, accurate measurement of the PM figure error - the segment motions that cause the PM figure to deviate from its nominal state.

Only the 6 global DOFs - the combined global tilt, twist, piston and translation of the PM as a coordinated whole - are not measured by the IDS, as they do not result in any segment edge displacements. These are primarily affected by gravity sag, incurred by changing the elevation angle of the telescope, and can be calibrated to high precision and compensated in a feed-forward, look-up table control, as discussed later in this report.
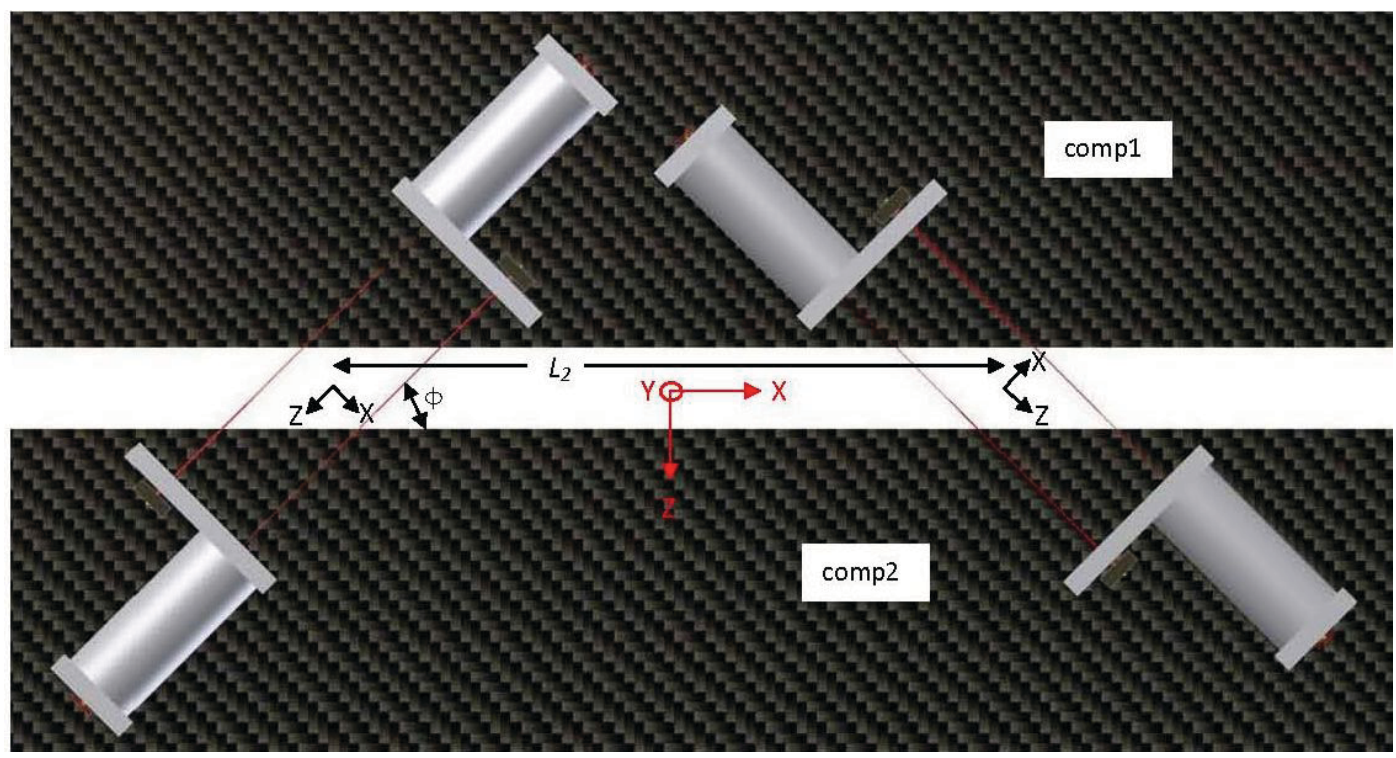

Figure 7. Two IDS sensor pairs along a segment edge.

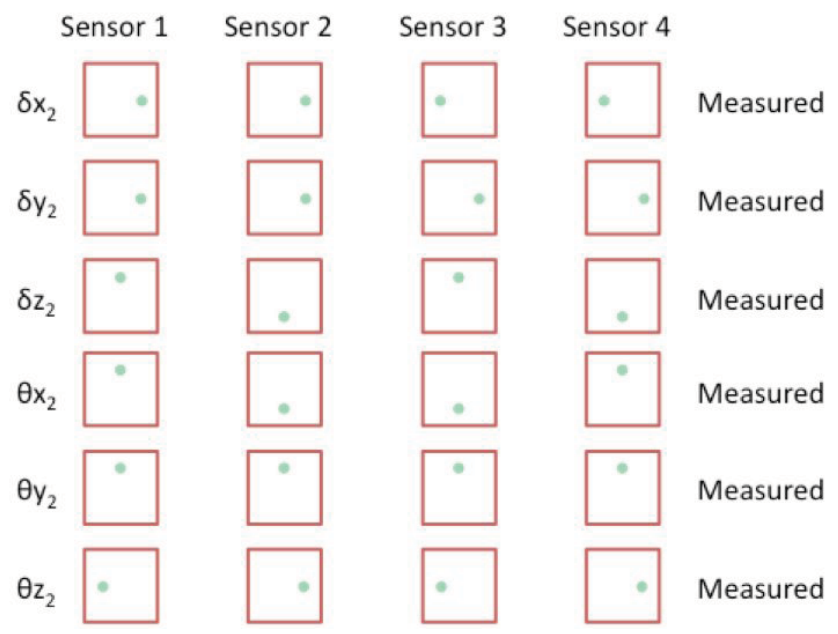

Figure 8. Measurements made by 2 IDS sensor pairs fully observe the 6 relative motions between the 2 adjacent segments (the lower segment moves relative to the upper).

For our CCAT WFC studies, we have chosen the sensor configuration illustrated in Figure 9, which shows the location of each sensor pair on the PM. For the CCAT primary mirror, with 162 segments, there are 624 sensor pairs, each sensing in 4 axes, for a total of 2496 measurements. Each segment has between 6 and 9 sensor heads, with most segments having 8 . These measurements allow full observability of the 968 mirror figure degrees of freedom - all but 
the 6 global rigid body motions of the primary mirror. As we will describe in later sections, this configuration meets current CCAT performance requirements.

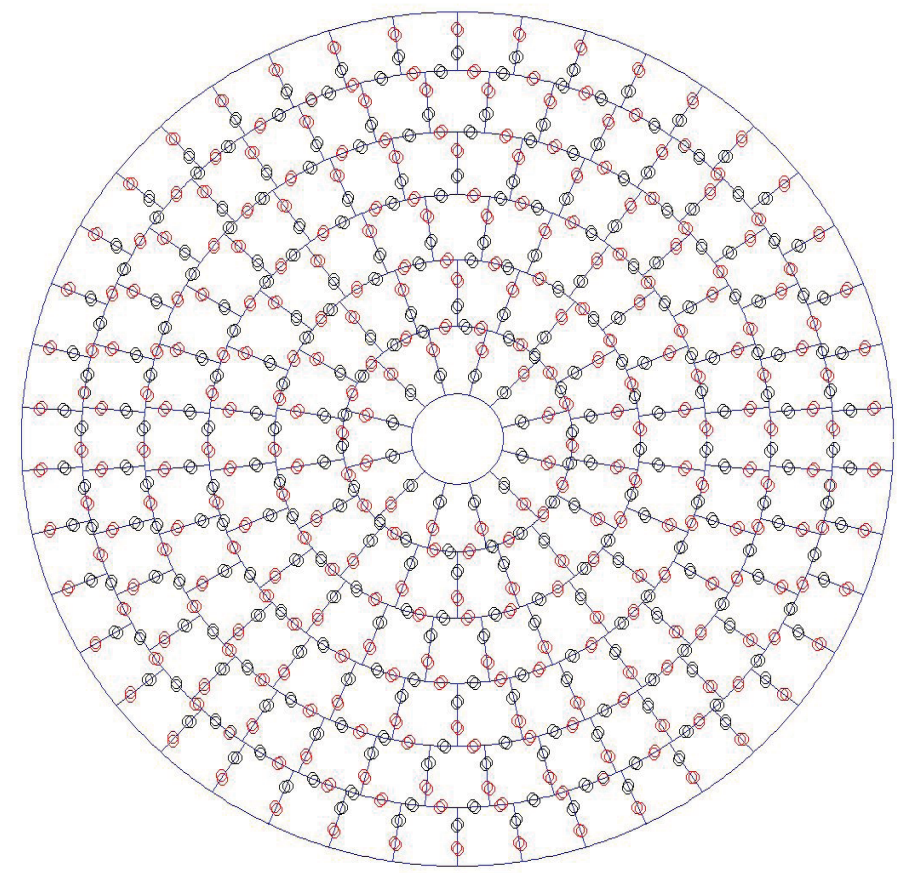

Figure 9. CCAT IDS locations. The black circles indicate sensor pairs oriented at $-45^{\circ}$ to the gap axis, and the red circles those that are oriented at $+45^{\circ}$.

\section{Wavefront Control System Overview}

This section provides a top-level overview of the CCAT WFC system, defining its basic elements, operational modes, and signal flow, to set the context for the derivation of the controller elements presented in the succeeding sections. Then the current baseline CCAT Error Tree is presented, to set performance objectives for the control system.

WFC elements. The main WFC elements are sketched in the block diagram form of Figure 10, which also shows signal flow at the highest level. The telescope "plant" consists of: the telescope structure, including the optics; the pointing actuators - gimbals which steer the telescope to point at science targets, and which change the direction of gravity relative to the telescope; the PM and SM actuators; the Optical Edge Sensors; and one or more cameras that can be used to sense the telescope WF.

The plant is subject to disturbances: variable gravity sag (relative to the telescope structure), which occurs as the telescope elevation angle changes; environmental temperature changes; and vibrations from internal and external sources. The vibration sources will include wind loading, the bearings and motors of the pointing system, the actuators, any fans or other moving equipment on the telescope, all as amplified or attenuated by the structural dynamics. The thermal disturbances include diurnal and seasonal temperature cycles, and internal heat sources such as electronics boxes.

The electronics that execute the sensing and control functions will be distributed according to function. Segment-level functions include reading IDS sensors and computing centroids, driving actuator motors, and reading auxiliary sensors such as accelerometers and thermal sensors. These functions can be handled with electronics located near each segment. 


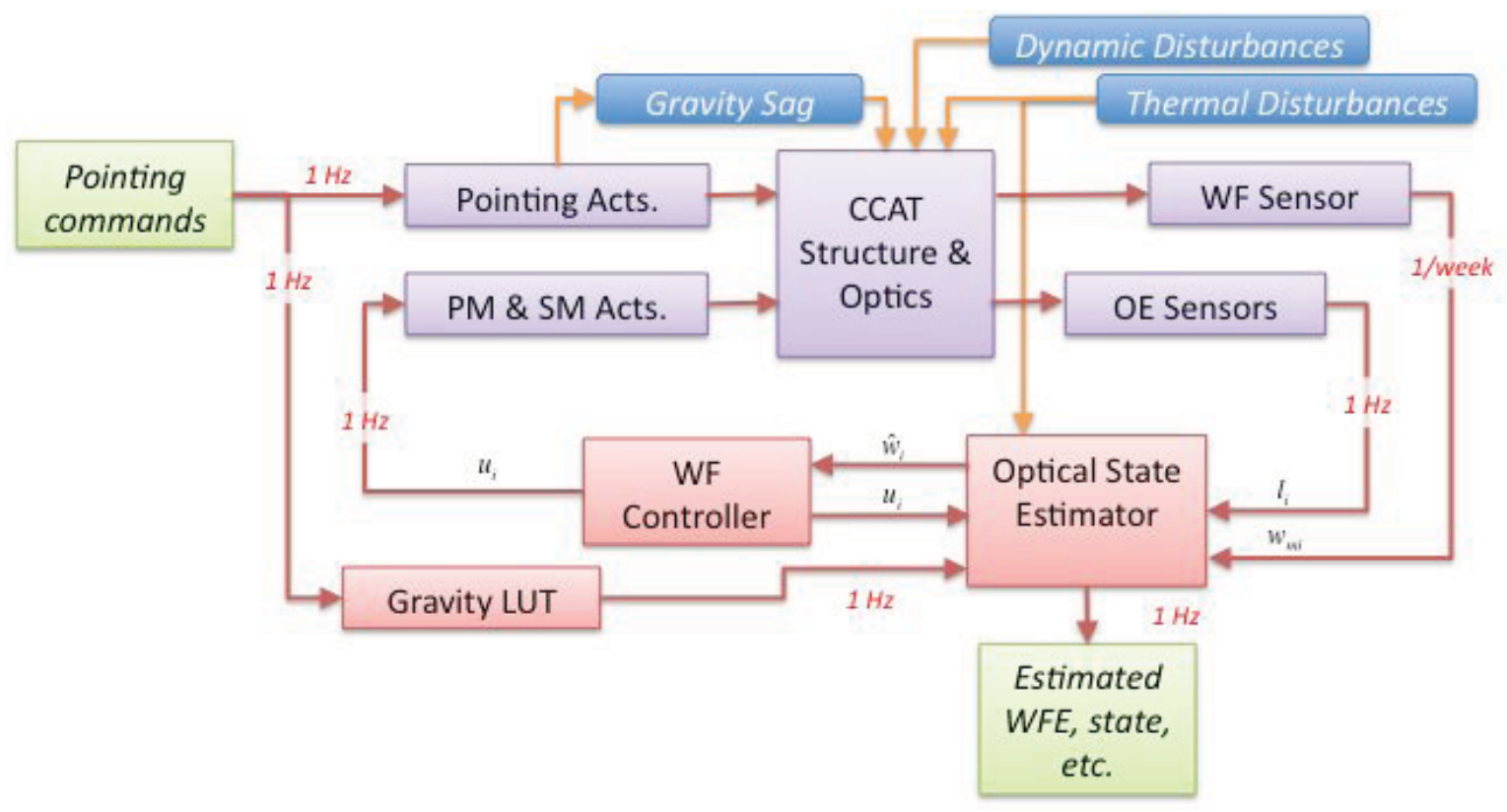

Figure 10. CCAT overall WFC system block diagram. Here purple boxes represent the plant to be controlled; blue boxes represent disturbances; red boxes represent major control elements; and green boxes represent operator interfaces.

Higher-level functions combine all of the IDS centroids, together with pointing and temperature information, to estimate the optical state and compute new actuator controls. These functions - the Optical State Estimator (OSE), the WF Controller, and feed-forward of gravity and possibly thermal information to the segment and SM actuators - will be executed by a central control computer.

User interfaces will include operator pointing commands, operator mode selections, and other functional settings and commands. Outputs from the OSE will include estimated WFE, estimated state, actuator positions, and other useful items for control diagnosis and display.

Wavefront Maintenance Mode. The most basic control loop, Maintenance Mode feeds back the IDS Optical Edge Sensor signals at $1 \mathrm{~Hz}$, to keep the PM segments aligned using the segment actuators. As will be described, the criterion for alignment is minimum WFE. This criterion is used by the OSE and WF Controller to choose the 3 actuator settings per segment that best compensate the WF effects of the measured 6DOF of disturbance. The Maintenance Mode controller requires occasional calibration of the reference WF - this is done using WF Sensing, as part of the Wavefront Initialization Mode.

Wavefront Initialization Mode uses direct WF sensing to measure and correct the total telescope system WFE, establishing the minimum-WFE reference condition for Maintenance Mode. As is discussed in [2], there are multiple WF sensing approaches that can be used. High-resolution submillimeter WFS, in either the image plane or the pupil plane, represents the most direct measurement approach, but is limited by lack of availability of sufficiently bright calibration sources. Lower-resolution submillimeter WFS, is more feasible with dim sources, and should prove useful for identifying SM/PM global mode alignment errors. Shorter wavelength IR WFS using a Keck Phasing Camera-type approach, which uses white-light interferometry over small patches of each segment, has many more calibration sources, but would require prohibitively expensive IR-quality PM segments and secondary mirror. The approach is to use imageplane submillimeter shearing interferometry, following the classic technique of Serabyn [10].

Feed-forward Gravity Look-Up Table control. The IDS measurements do not observe the global PM motions, only motions of the segments with respect to each other. The IDS also does not measure SM motions, and yet the position and angle of the SM relative to the PM - the collimation of the telescope - affects wavefront error and image motion to a high degree. Furthermore, telescope collimation is strongly driven by gravity sag variation as the telescope changes its elevation angle during notrmal observations. 
There are multiple options for countering gravity sag effects. One is to add measurements of the PM/SM collimation, using an optical collimator or a laser metrology system, perhaps. Another is to use a Look-Up Table (LUT) that records the amount of decollimation that occurs when the telescope changes its elevation angle. Since the CCAT structure is likely to be highly linear and repeatable, the gravity sag prediction should be accurate to within a few percent, and should provide an adequate solution for gravity sag variation effects. For this implementation:

- The LUT produces SM commands in 5 DOF, based on the predictions of a FEM, and are determined as the global rigid-body motions that minimize WFE from the predicted sag.

- The LUT may also produce "segment global rigid-body" commands to move the PM segments in a coordinated global tip, tilt and piston motion. These motions are not seen by the IDS, as they occur in modes orthogonal to the $\partial l / \partial x$ matrix.

- The FEM will be updated by laser tracker measurements at multiple elevation angle positions prior to first light.

- The FEM will be updated by WF Sensing during WF Initialization control at commissioning and periodically thereafter.

Global temperature changes also have a systematic and repeatable impact on the telescope global WF and pointing. These changes are not expected to require WF control compensation at this time. If they turn out to be larger than currently predicted, they could be partially compensated using feed-forward control based on temperature measurements; alternatively, thermal modes could be added to the estimator, and they could be corrected using this augmented state feedback.

Commissioning Phase Operations will begin once CCAT is assembled and mechanically aligned. The initial configuration of the telescope will occur with the telescope pointing to zenith. Alignments will be made using laser trackers monitoring the position of fiducials mounted to the critical optics: the PM segments; the SM, the Fold Mirror and instrument optical bench or mounting ring. Expected precision of the initial alignments, based on [6], is 50 um and 50 urad.

Commissioning will be performed with the Wavefront Maintenance Control turned on, so that the segments will be under closed-loop servo control, allowing repeatable and consistent positioning of the PM segments.

Wavefront Error Tree. When first light occurs, Wavefront Initialization Control will be performed to reduce the WFE effects of the original misalignments. WF Initialization occurs with the telescope observing a bright calibration source, nominally Mars. A WF Sensor directly measures the total system WF, including effects from the PM and other optics. WF controls are applied to compensate for the full optical system WF error. With multiple iterations of WFS\&C, the WFE will be reduced to a small value, at least for that particular gravity and thermal condition.

To track WF error performance, the CCAT project has developed an Error Tree model of the telescope WF error. This takes the form of an error budget [7], summarized spreadsheet form in Figure 11. The subsystem errors are the contribution of WF errors to the total WFE, as computed by analysis, based on component specifications.

The "Model Only" column of the spreadsheet in Figure 11 rolls up the errors that are considered in the WF Control models we discuss in this report. These numbers, 5.36 um total telescope WFE at the top, and lower-level numbers, provide performance targets for control system development.

The allocation of errors given in the Error Tree is conservative, in the following ways:

- No WF correction of non-Primary Mirror related errors is assumed. In fact, performing occasional WF sensing will allow some correction of SM and TM WF errors, reducing their contribution to the overall WFE. WF sensing and WFS-based control is discussed below, in Sections 8 and 9.

- A conservative WF control approach is assumed, with fixed-gain estimators used, rather than better-performing (but slightly more complex) variable-gain estimators. Current predictions show that the fixed-gain estimators provide adequate performance; variable gain estimators could be adopted if margins shrink in the future.

- No effort is made to estimate or control systematic errors due to measurable thermally-driven deformations. "Cupping" errors due to thermal gradients, for instance, could be modeled and controlled, but current performance predictions do not show that this is necessary. 


\begin{tabular}{|c|c|c|c|c|}
\hline \multicolumn{5}{|c|}{ CCAT WFE Tree } \\
\hline System & $\begin{array}{l}\text { Non } \\
\text { Component } \\
\text { WFE (um } \\
\text { RMS) }\end{array}$ & $\begin{array}{l}\text { ninal } \\
\text { System WFE } \\
\text { (um RMS) }\end{array}$ & $\begin{array}{l}\text { Mode } \\
\\
\text { Component } \\
\text { WFE (um } \\
\text { RMS) }\end{array}$ & $\begin{array}{l}\text { only } \\
\text { System } \\
\text { WFE (um } \\
\text { RMS) }\end{array}$ \\
\hline $\begin{array}{c}\text { Telescope Totals } \\
\text { Secondary } \\
\text { Tertiary } \\
\text { Instrument } \\
\text { Mount } \\
\text { Design }\end{array}$ & $\begin{array}{r}12.56 \\
8.9 \\
0.1 \\
0 \\
8.78\end{array}$ & 21.55 & $\begin{array}{l}0 \\
0 \\
0 \\
0 \\
0\end{array}$ & 5.32 \\
\hline $\begin{array}{l}2 \text { Primary } \\
\text { Thermal Gradient } \\
\text { Actuator heating } \\
\text { Actuation error } \\
\text { Acceleration } \\
\text { Subframe } \\
\text { Truss } \\
\text { Tile }\end{array}$ & $\begin{array}{r}0.02 \\
0.006 \\
0 \\
2.94 \\
0.314 \\
3.1 \\
6.6\end{array}$ & 12.27 & $\begin{array}{r}0.02 \\
0 \\
0 \\
0 \\
0.314 \\
3.1 \\
0\end{array}$ & 5.32 \\
\hline $\begin{array}{l}\text { 3 Control } \\
\text { Gravity } \\
\text { Thermal Gradient } \\
\text { Actuator heating } \\
\text { dCTE cupping } \\
\text { Sensor noise }\end{array}$ & $\begin{array}{r} \\
2.5 \\
1.38 \\
1.38 \\
7.86 \\
0.7\end{array}$ & 9.41 & $\begin{array}{r}2.5 \\
1.38 \\
0 \\
0 \\
0.7\end{array}$ & 4.32 \\
\hline $\begin{array}{l}4 \text { Estimation } \\
\text { LUT error } \\
\text { Model error } \\
\text { Latency / Drift }\end{array}$ & $\begin{array}{r}3 \\
2.5 \\
1\end{array}$ & 4.03 & $\begin{array}{l}3 \\
0 \\
1\end{array}$ & 3.16 \\
\hline
\end{tabular}

Figure 11. CCAT Error Tree Spreadsheet

\section{Linearized CCAT Model}

The computer models described in the previous section - the MACOS optics model and the NASTRAN structures model - can be used to generate linearized, matrix models of the CCAT structure, optics, sensors and actuators. These linearized models are highly accurate over the small perturbations that are typical of a telescope, especially one with an active WFC system. These models are directly embedded into the estimator and controller, as described in Section 6.

The models are based on an Optical State Vector $x_{i}$, which captures all expected optical perturbations, as driven by the various disturbances, noise and errors inherent to the telescope, and as mitigated by the WFC system. This is based on the theory of reference [8]. We treat the telescope as a multi-stage, evolving system, driven by noise, and by systematic disturbances such as gravity sag and thermal deformations. This model, used to derive the WFC system control gains, does not explicitly include structural or atmospheric dynamics. A full dynamical model will also be used to test the controls in a richer disturbance environment.

Optical state vector $\boldsymbol{x}_{i}$. The full optical state vector at the $i^{\text {th }}$ time interval includes both rigid body perturbations $x_{R i}$ and surface deformations $x_{D i}$ of all of the optics into a single vector:

$$
x_{i}=\left[\begin{array}{l}
x_{R i} \\
x_{D i}
\end{array}\right]
$$

The "rigid-body optical state vector" $x_{R i}$ contains the rigid body perturbations of all of the optics, defined as small $\mathrm{x}, \mathrm{y}$ and $\mathrm{z}$ translations and rotations, evaluated at the $i^{\text {th }}$ time interval, in local coordinates: 6 Degrees of Freedom (DOF) per optical element. These perturbations are relative to the nominal state of the telescope: the ideal, as-designed telescope will have $x_{R i}=0$. For CCAT, with 162 PM segments, the SM, and treating the FM and detector as a single structure, $x_{R i}$ is a vector of dimension $948 \times 1$. The state is defined in coordinates local to each optic, centered at the pole of the optic, oriented along the local radial, tangential and normal directions, relative to the commanded global telescope pointing.

The "deformation optical state vector," $x_{D i}$ contains surface deformation maps, capturing the effects of individual optical distortions, due to fabrication errors, thermal deformations, and other slow or static effects. To first order, $x_{D i}$ will not be affected by the rigid body actuators, but there may be some unintended coupling if the actuator attachments are too stiff. 
Optical state transition equation. The state $x_{i}$, and especially the rigid-body component of the state $x_{R i}$, changes in time, due to actuations, changes in the direction of gravity, thermal deformations, and the lumped effects of structural dynamics and structural nonlinearities, which will be treated here as a process noise $\xi_{i}$ : a random change in the state elements themselves. The state transition equation governs the evolution of the full state $x$ from time interval $i$ - 1 to time interval $i$. A time history of the state can be computed by "integrating" $x$ forward in time, from a defined initial condition $x_{0}$, to an arbitrary final time.

The actual time interval represented by $i$ is chosen to be the shortest time interval used in the WFC system, generally the time interval between subsequent edge sensor measurements, nominally $0.5 \mathrm{sec}$.

Control actions are denoted $u$ : a vector where each element is the command sent to a particular actuator. Each segment is equipped with 3 actuators, which, in combination, control its tip, tilt and piston in the same local coordinate frames, and the SM is equipped with 6 actuators. It will be convenient to book-keep the SM actuations separately, as $u_{S M}$.

The coupling of $u$ to $x$ is by a kinematics matrix $\partial x / \partial u$. The true kinematics of the actuators will depend on currently unspecified design details, so our current model treats each element of $u$ as directly actuating a segment DOF: tip, tilt, or piston. The matrix $\partial x / \partial u$ is therefore currently a selection matrix, whose columns are taken from the columns of a 968 square identity matrix; its dimension is $3 \times 162+6$, or 492 , by 968 .

The dynamics of the CCAT structure are not explicitly included in the control derivation model, though ultimately they will be included in a more detailed system performance simulation: the "truth model." For control design purposes it is sufficient to envelope the behavior of the CCAT structure, using a process noise term $\xi$ to introduce random state changes that represent the integrated effects of noise-driven dynamics over the time interval between control steps.

Systematic structural modes, such as those due to changes in temperature, or changes in telescope pointing (which change gravity loading), are represented as coupled disturbance modes $T$. These include thermal modes of the segments, thermal modes of the telescope structures, the effects of gravity as the telescope pointing changes, global pointing errors, and other global effects.

The state transition equation sums all of these changes to calculate the new state:

$$
x_{i}=x_{i-1}+\frac{\partial x}{\partial u} u_{i}+\frac{\partial x}{\partial u_{S M}} u_{S M i}+\frac{\partial x}{\partial T} T_{i}+\xi_{i}
$$

That is, the state at the $i^{\text {th }}$ time interval is equal to the state at the $i-1^{\text {th }}$ time interval, plus changes induced by actuator motions, changes due to disturbance modes, and changes due to the process noise $\xi$.

Wavefront and pointing equations. Changes in the state $x$ have optical effects: wavefront $w$ degrades; the telescope pointing $c$ wanders. The fully-nonlinear CCAT MACOS model computes these optical effects by perturbing the position and shape of each optic, and then tracing rays through the optical system, from a star at infinity, past each optic to the detector. One result is an OPD or WFE map: a map of the wavefront across the pupil, evaluated at an exit pupil conjugate to the PM. Another result is the Point Spread Function: the star image as recorded at the focal plane.

The wavefront $w$ is the vectorized form of the OPD matrix, generated by stacking the non-zero elements of the columns of the OPD matrix into a single long vector. CCAT OPD matrices have dimension 512 by 512 , and the $w$ vector has dimension 17,683 by 1 . Simple mapping functions ( $\mathrm{m} 2 \mathrm{v}$ and $\mathrm{v} 2 \mathrm{~m}$ ) allow conversion of OPD to $w$ and vice versa. The RMS Wavefront Error (WFE) is computed as the RMS of $w_{i}$ :

$$
W F E_{i}=\operatorname{rms}\left(w_{i}\right)
$$

MACOS can be used to compute sensitivity matrices $\partial w / \partial x$, which quantify the first-order influence on $w$ of each element of $x$. MACOS can do this using analytical forms for many element types, or by numerical differentiation. Each column of $\partial w / \partial x$ represents the effect on $w$ of a unit excursion in the corresponding entry in $x$; the product of $\partial w / \partial x$ and $x$ is the total effect on $w$ of all of the perturbations in $x$. So, at the time interval $i$, the total $w$ is:

$$
w_{i}=\frac{\partial w}{\partial x} x_{i}+w_{A t m}+w_{0}
$$


The WF $w$ is equal to the nominal WF $w_{0}$ plus the optical effects of the perturbations $x$, plus the effects of atmospheric turbulence $w_{A t m}$ (small in the submillimeter); similarly for the pointing $c$. Here MACOS is used to generate the optical sensitivities $\partial w / \partial x$ and $\partial c / \partial x$, following the theory of [8]. This provides linear optical models that predict the WFE and pointing error using matrix operations rather than a full ray-trace; such models are highly accurate for the small state excursions typical of a controlled telescope. The RMS Wavefront Error (WFE) is computed as the RMS of $w_{i}$.

Wavefront measurement equation. Changes in the state $x$ also impact the outputs of the measurements that are used for control. As will be described, direct WF measurement will be used in the early phases of control to phase the telescope against an external optical reference and calibrate the WFC edge sensors; it will be used periodically thereafter to check the phasing and determine new set points for the WFC edge sensors. In this paper we consider 3 types of measurement. The first is a direct measurement of the WFE, which can be obtained by WF sensing. The WF measurement equation takes the form (assuming fully unwrapped phase):

$$
w_{m i}=w_{i}+\delta w_{i}=\frac{\partial w}{\partial x} x_{i}+w_{A t m}+w_{0}+\delta w
$$

Here $\delta w$ is the wavefront measurement noise.

Edge sensor measurement equation. The second type of measurement is made using the IDS optical edge sensors. Translational or rotational motions of one segment relative to the other cause lateral displacement of the light beam, which is measured by the CCD in 2 axes: in the " $z$ " or local surface normal direction; and "y," parallel to the mirror surface, as described in [12] and [13]. Optical edge sensors are placed as shown in Figure 8, with approximately 2 sensors per segment-to-segment edge, and a total of 624 sensors, each sensing in 2 axes for a total of 1248 measurements. Design parameters governing edge sensor performance include the mounting position below the surface and the radial position of each sensor.

The edge sensor measurement equation takes the form:

$$
l_{i}=\frac{\partial l}{\partial x} x_{i}+l_{0}+\delta l_{i}
$$

Here the $\partial l / \partial x$ sensitivities capture the kinematics of the sensors. The $l_{0}$ vector represents the static offset of the edge sensors, which can be calibrated, and $\delta l$ is the edge sensor measurement noise. Optical edge sensor measurements will be made at a frequency of 0.1 to $1 \mathrm{~Hz}$.

Modal analysis. The question arises, are the IDS measurements sufficient to observe all of the optically significant motions of the telescope, or are there modes that cannot be accurately measured? The answer is determined by singular value decomposition of the $\partial l / \partial x$ matrix:

$$
(U, S, V)=\operatorname{svd}\left(\frac{\partial l}{\partial x}\right)
$$

Modes $j$ with large singular values $S_{j}$ will be easily measured by the IDS; modes with very small or zero singular values $S_{j}$ will not be measured by the IDS. In fact, as illustrated in Figure 14, there are 966 modes strong modes, covering a dynamic range of only 161 - these modes are well measured by the IDS. There are also 6 weak modes, with singular values $10^{5}$ times smaller than the largest. 

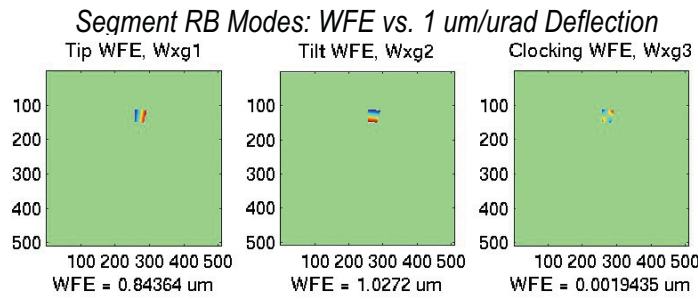

Edae Sensor Response to Seament RB
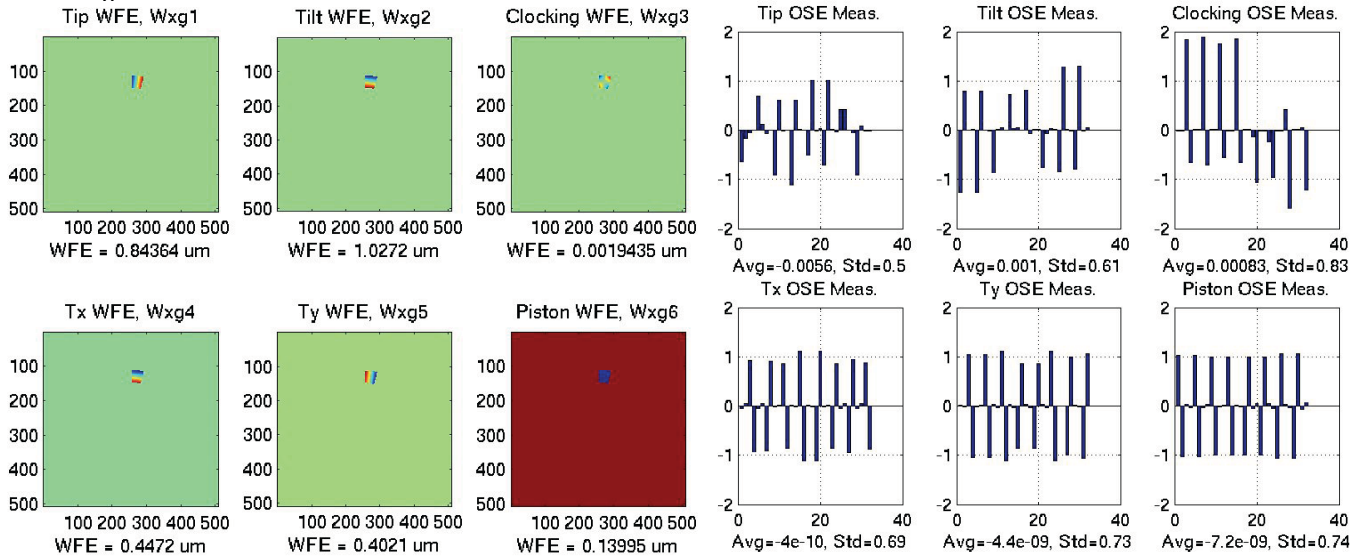

Figure 12. Wavefront (left) and affected IDS measurements (right) due to segment 42 rigid body motion.
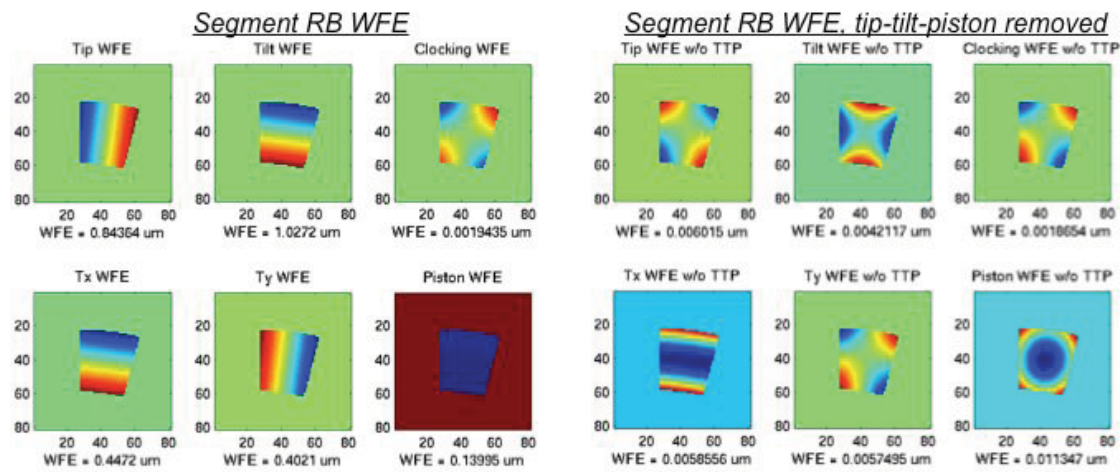

Figure 13. Zoomed-in plot of wavefront (left), and tip-tilt-piston removed WF (right), due to rigid body motion of segment 42.

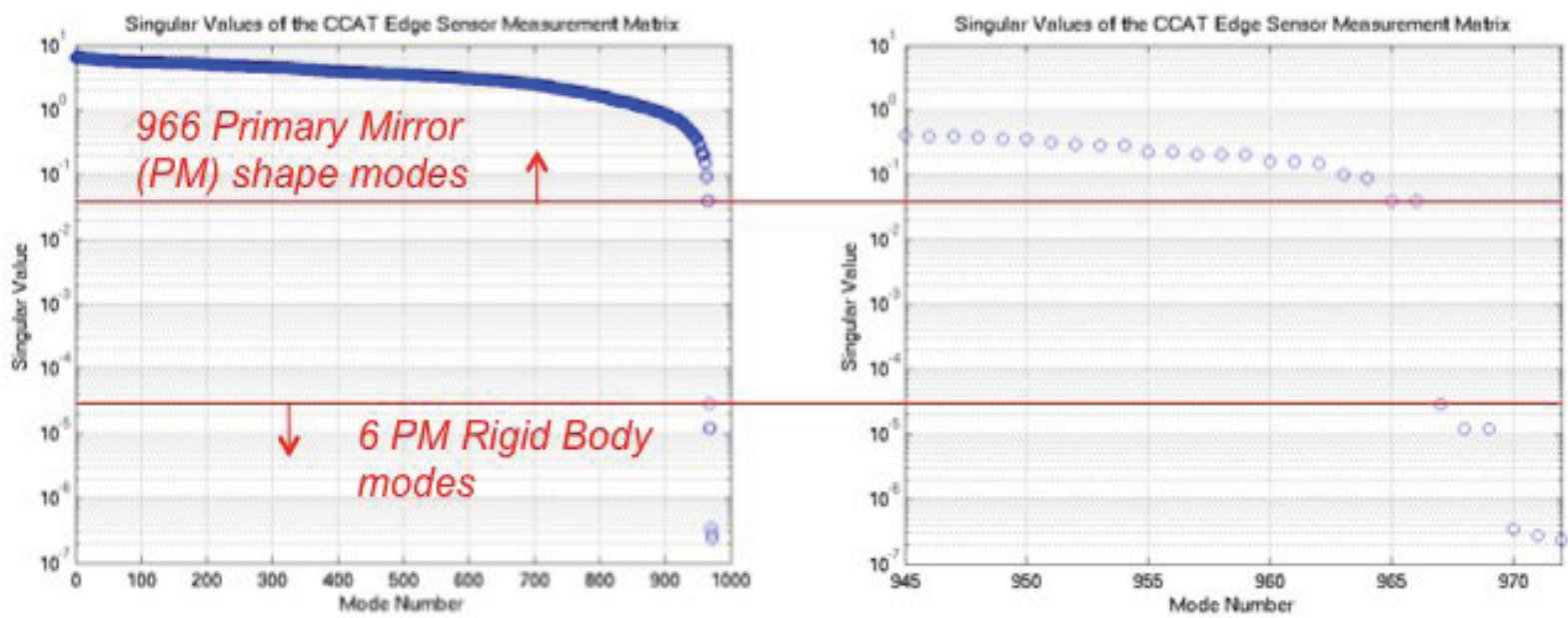

Figure 14. Singular values $S_{j}$ of the IDS measurement matrix $\partial l / \partial x$, showing that there are 6 near-zero singular values, corresponding to unmeasurable modes of $x$. Plot on the right shows the lowest singular values in more detail.

The optical impact of a mode $j$ can be computed from the $j^{\text {th }}$ column of $U$ (denoted $U_{j}$ ), as: 


$$
w_{j}=\frac{\partial w}{\partial x} U_{j}
$$

The 6 weakest modes are the tip-tilt-piston-removed residue of the global rigid body modes: the coordinated 6DOF motions of all of the PM segments together as a single rigid body.

It would seem reasonable to expect that a PM global tilt would produce a pure tilt of the WF, with no residual WFE after tilt is removed, but this is not the case. Tilt or lateral displacement of the PM as a whole acts to decollimate the telescope, breaking the precise alignment of the PM with the SM and focal plane, leaving a classically comatic wavefront error. This means the the 6 weakest modes have significant WFE impact.

In summary, modal analysis shows:

- The 966 non-global modes of motion of the PM segments are well measured by the IDS.

- The 6 global PM rigid-body modes - coordinated tip, tilt, twist, $\mathrm{x}$ - and $\mathrm{y}$ - translation, and piston of the PM as a coordinated whole - are not measured by the IDS.

- The 6 global PM modes induce coma, focus, tilt and astigmatism WFE, and need to be compensated by other means - by collimation measurements, or by feed-forward methods

\section{Wavefront Estimation and Control}

This section derives the Optical State Estimator (OSE), which is used to estimate the optical state and predict the wavefront based on IDS measurements; and the Wavefront Controller, which uses the predicted wavefront in a control law that minimizes wavefront error (WFE). This approach makes it possible to compensate for effects that are measured but can't be directly controlled, such as in-plane translation of the segments. The result is vastly improved performance compared to approaches that merely seek to null errors in the controlled DOFs alone.

The OSE uses a Kalman filter to continuously estimate the CCAT optical state. The Kalman filter provides a formally optimal method for balancing measurement noise and state knowledge errors, by recomputing its feedback gains each time step, based on evolving estimates of the statistics the component errors. This gives it the ability to adjust to varying conditions, but at a computational cost. Simplifications of the full OSE are therefore considered, that do not update the estimator gains at each control step, but that do provide near-optimal performance for the expected noise and error conditions.

\subsection{Optical State Estimator}

The optical edge sensor measurements $l$ (and $w$ when available), can be used to estimate the optical state $x$ of the telescope at any particular time; and that estimate can be used to compute new controls $u$. This is the function of the OSE: to take in all of the available data, and to process it to determine the underlying optical state $x$ of the telescope. The data that is continuously available includes the measurements $l$ with covariance $L$; pointing measurements $c$ with covariance $C$; actuations $u_{R}$ and $u_{S M}$ and their error covariances $U_{R}$ and $U_{S M}$; and the covariance of the process noise, $\Xi$.

The OSE combines this data with a prediction of the state based on the previous state estimate to carry over information from earlier measurements. The formally optimal way to do this is by Kalman Filter techniques: weighted least-squares, where the estimate weights the measurements and the prior knowledge using the covariance matrices associated with each term to find the most likely state estimate [14], [15].

The Kalman Filter (KF) is a predictor-corrector estimator, where at each measurement we make a prediction of the measurement, based on the information we had at the last measurement, and compare that to the actual current measurement. The difference of the predicted and actual measurements, termed the innovation, is multiplied by a gain matrix and fed back to update the estimate. The gains are weighted by the covariances of the measurement and priorestimate errors, so as to achieve an optimal blending of prior and current information.

The prediction is made by taking the estimate computed at the preceding time step and simply projecting it forward in time using Eq. 2. We know the value of the control we commanded, so that goes into the prediction as well. The predicted measurement is then calculated by projecting the predicted state to the WF space using Eq. 3. Denoting the

predicted state at time $i$ with an over-bar as $\bar{x}_{i}$, and the estimated state with a hat, as $\hat{x}_{i}$, the predicted state is: 


$$
\bar{x}_{i+1}=\hat{x}_{i}+\frac{\partial x}{\partial u} u_{i}
$$

The predicted measurement is thus:

$$
\bar{l}_{i}=\frac{\partial l}{\partial x} \bar{x}_{i}
$$

The new state estimate is simply the prediction plus the innovation weighted by a gain matrix $K_{i}$ :

$$
\hat{x}_{i}=\bar{x}_{i}+K_{i}\left(l_{i}-\bar{l}_{i}\right)
$$

The gain balances the contribution of the innovation to the current estimate as the ratio of the error in the predicted state, as captured in the predicted-state covariance $P_{i}$, to the error in the measurement projected back to the state space via the transpose of the measurement matrix. We treat the actuator commands as known signals with a measurement error equal to the actuation noise projected to the WF space. The error in the measurement is the covariance matrix $R_{i}$, which includes the WF measurement error and the actuation error $\delta_{u}$. The gain is:

$$
K_{i}=P_{i}\left(\frac{\partial l}{\partial x}\right)^{T} R_{i}^{-1}
$$

Note that the gain matrix $K_{i}$ changes with every time step, which can impose a large computational burden on the OSE. In practice, however, $K$ will quickly approach a nearly static steady state, so that it is possible to precompute a set of $K_{i}$ matrices for the various different control conditions, and use them while those conditions obtain, as discussed later.

The measurement error covariance, which captures the uncertainty in both the actuation and the measurement, is:

$$
R_{i}=\frac{\partial l}{\partial x} \frac{\partial x}{\partial u} U_{i}\left(\frac{\partial l}{\partial x} \frac{\partial x}{\partial u}\right)^{T}+L_{i}
$$

The error in the estimate is captured by its covariance $P_{i}$, which combines the measurement and predicted-state error covariances $R_{i}$ and $M_{i}$ in the state space, as:

$$
P_{i}=\left[M_{i}^{-1}+\left(\frac{\partial l}{\partial x}\right)^{T} R_{i}^{-1} \frac{\partial l}{\partial x}\right]^{-1}=\operatorname{cov}\left(\tilde{x}_{i}\right)
$$

The covariance of the predicted state is computed based on the covariances of the error in the previous estimate, projected forward to the current time:

$$
M_{i+1}=P_{i}+\frac{\partial x}{\partial u} U_{i}\left(\frac{\partial x}{\partial u}\right)^{T}+\Xi_{i}
$$

Implementation of the Kalman update (Eq. 16), after substitutions, can take a simpler form:

$$
\hat{x}_{i}=\left(I-K_{i} \frac{\partial l}{\partial x}\right) \hat{x}_{i-1}+\left(I-K_{i} \frac{\partial l}{\partial x}\right) \frac{\partial x}{\partial u} u_{i-1}+K_{i} l_{i}
$$

For gaussian normal random processes, the KF estimator of Eqs. $19-24$ produces optimal estimates, in the sense that they make the best possible use of the available information, producing estimates with minimal error. In fact, the KF defines the Cramer-Rao bound for such processes [14]. In practical problems the KF assumptions are not always 
precisely met. The statistics of the processes may not be normal or stationary, the plant may not be truly linear - but for small deviations from the assumptions underlying the KF, the KF provides an excellent and practical tool.

Equations 17-24 define the KF updates provided by the optical edge sensors, which occur at every cycle of the OSE. Other measurements, namely the WF measurements $w$, or pointing measurements if made, are incorporated in the same way, but at different times. The WF measurements will be made perhaps once a month, for example. When such measurements are available, they can be used to update the state $x$ in exactly the same way [2].

\subsection{Wavefront Controller}

Wavefront control using WF measurements. The WFE can be controlled by directly measuring $w$ using a WF Sensor, and then using the measured $w$ to compute new control values that minimize WFE. WF measurements measure all of the contributors to WF error, not just the PM segment errors measured by the IDS. The resulting WFC can compensate all of those errors, including SM WFE, TM WFE, and PM global modes. WF measurements are available to CCAT only very occasionally, during commissioning, and during calibration opportunities that may follow thereafter, when a suitable calibration source (Mars, e.g.) is available.

The cost function for control using WF measurements is:

$$
\min _{u} J=w^{T} w+c_{u} u^{T} u
$$

There are 2 terms here, balanced by a scalar coefficient $c_{u}$. The first term is the square of the WF; the second is the square of the control effort between the $i^{\text {th }}$ and the $i-1^{\text {th }}$ iteration. The coefficient can be tuned to balance the control effort against the WFE, a useful knob to turn in modulating the control response. The control law that satisfies this cost function is:

$$
u_{i}=-\left[c_{u} I+\left(\frac{\partial w}{\partial x} \frac{\partial x}{\partial u}\right)^{T} \frac{\partial w}{\partial x} \frac{\partial x}{\partial u}\right]^{-1}\left(\frac{\partial w}{\partial x} \frac{\partial x}{\partial u}\right)^{T} w_{i}
$$

WFS measurements directly measure the WF and can be used without the OSE to perform control as per Eq. 32 - or they can be used to update the estimated state using the OSE, and then control can be performed using the estimated state. The latter approach allows the information in the WF measurement to be used to update the OSE, improving all future state estimates; it also utilizes the noise filtering functions of the OSE; and it should be preferred.

Wavefront control using the estimated state. The IDS edge sensors do not measure the WF, but they can be used to predict the WF, using the continuously-updated estimated state provided by the OSE. This permits use of a minimumWFE control law, which has important performance advantages. At any time interval $i$, the predicted WF is:

$$
\bar{w}_{i}=\frac{\partial w}{\partial x} \hat{x}_{i}
$$

Substituting for $w_{i}$ in Eq. 32, the "Wavefront Compensation" control law is:

$$
u_{i}=-G \hat{x}_{i}
$$

The gain matrix $G$ is:

$$
G=\left[c_{u} I+\left(\frac{\partial w}{\partial x} \frac{\partial x}{\partial u}\right)^{T} \frac{\partial w}{\partial x} \frac{\partial x}{\partial u}\right]^{-1}\left(\frac{\partial w}{\partial x} \frac{\partial x}{\partial u}\right)^{T} \frac{\partial w}{\partial x}
$$


Using the estimated state as the basis for CCAT control allows the controller to use actuation in the controlled DOFs to mitigate the optical effects of errors that are incurred in non-actuated DOFs. An example case is the lateral translation of a segment. Such a motion occurs when the base structure expands, and the consequence is a small but significant WFE, as the segment moves off of the ideal surface. This error occurs in a direction mechanically - but not optically orthogonal to the 3DOFs along which the actuators act. By moving the segment in piston and tilt, the optical effects of this displacement can be largely corrected.

\subsection{Fixed-Gain OSE.}

The computations required to compute the full OSE gains at each time step are large: a $n_{x}$-by- $n_{x}$ add to compute $M_{i+1}$ (Eq. 23); a $n_{x}$-by- $n_{x}$ add and inversion to compute $P_{i}$, the covariance of the estimate error (Eq. 22); then a $n_{x}$-by- $n_{x}$-by- $n_{l}$ multiply to compute $K_{i}$ (Eq. 20). Performing these computations at a 1 or $2 \mathrm{~Hz}$ rate will require a reasonably powerful central control computer.

An alternative is to use a "fixed-gain," non-optimal version of the OSE. This reduces the computational burden for the central computer, but will incur a performance penalty. This penalty will be small in many cases, making the fixed-gain approach attractive. Fixed-gain controls can also use "gain scheduling," where different gain matrices can be computed ahead of time, and manually or automatically selected according to current conditions.

There are 3 main methods for computing the gains for a fixed-gain OSE. The first is simply to use a single-step approach, as described in [15]. This approach uses a single-step form of Eqs. 17-23, to determine gains based on $X_{0}$ (the initial state covariance) alone. It will perform near optimally when state errors are consistent with $X_{0}$, but less than optimally when $X_{i}$ differs from $X_{0}$. If $X_{0}$ is chosen to be large, to handle large initial conditions, the gains will favor the measurements over prior state knowledge. Then, when after several iterations the state estimate errors become small, the estimator will not put enough emphasis on the prior state knowledge. If measurement errors are very small, this will not be a serious problem: performance, while not optimal, should be good enough. On the other hand, if measurement errors are large, this approach will lead to degraded performance.

The second method for computing gains for fixed-gain OSE is simply to run the full OSE for a number of time steps, in representative conditions, and then record the $K$ matrix (Eq. 20) for use in the future. The number of time steps that are run before freezing the gains will affect the fixed-gain OSE response. If it is run until it reaches a steady state, where the estimate error covariance $P$ is essentially constant from time step to time step, the fixed-gain estimator response will be slow, as the state covariance $X_{i}$ will be small compared to the measurement covariance $L_{i}$. If it is run only a few time steps, it will likely favor the measurements over the prior statee information, leading to a faster response and a higher ultimate WFE floor. The user should experiment to find the best balance of response and ultimate noise floor.

The third method is to directly derive steady-state gains, assuming stationarity, as described in [2].

\section{Wavefront Control Performance Examples}

This section examines WF Maintenance Control performance against selected CCAT disturbances, such as gravity sag, IDS sensor error, and thermal deformations. It also compares performance of fixed-gain and variable gain Kalman filter OSE implementations.

\subsection{A simulated WF Maintenance Control example.}

To illustrate the Maintenance Control performance, consider the following simulated example, modeling recovery from a large initial error. Particular parameters used in this case are:

- Initial state error covariance: $X_{0}=\operatorname{diag}\left(\sigma_{x 0}{ }^{2}\right)$, with $\sigma_{x 0}=25 \mathrm{um}$ for translation states and $\sigma_{x 0}=25$ urad for rotational states.

- Initial state is computed using a uniform random number generator, to produce a single $n_{x}$-by-1 state vector whose statistics are consistent with the initial state covariance: $x_{0}=\sigma_{x 0} \times \operatorname{rand}\left(n_{x}, 1\right)$.

- Process noise: $\sigma_{\zeta}=0.01 \mathrm{um}$, and the process noise covariance matrix is $\Xi=\operatorname{diag}\left(\sigma_{\zeta}^{2}\right)$; individual realizations of noise $\zeta=\sigma_{\zeta} \times \operatorname{randn}\left(n_{x}, 1\right)$ are added to the state at each time step (Eq. 2).

- Measurement noise: $\sigma_{l}=0.1 \mathrm{um}$, and the measurement noise covariance matrix is $L=\operatorname{diag}\left(\sigma_{l}^{2}\right)$; individual realizations of noise $\delta_{l}=\sigma_{l} \times \operatorname{randn}\left(n_{l}, 1\right)$ are added to the measurements at each time step (Eq. 7). 
- Actuator noise: $\sigma_{u}=1 \mathrm{~nm}$ in this case, and the actuator noise covariance matrix is $U=\operatorname{diag}\left(\sigma_{u}{ }^{2}\right)$; individual realizations of noise are added to each actuation as $\delta_{l}=\sigma_{u} \times \operatorname{randn}\left(n_{l}, 1\right)$ (Eq. 2). Note that some time steps do not include actuations for some segments, and so no actuation noise is added to those segments (and the OSE gains do not include the actuation uncertainty terms).

- $\quad$ Control parameters $c_{u}$ and $c_{v}$ are set to 0 .

- The initial WF and the initial WF estimate is shown on Figure 15, both with and without global modes included. The WF estimate closely matches the total WF in this case, where there is little participation of unobservable global modes. The error drops sharply after a single control step (Figure 16), and then continues to drop more slowly, dropping below 0.3 um by the $22^{\text {nd }}$ step, as shown in Figure 17. Step-by-step WFE and estimated WFE performance is summarized on Figure 18. Note that measurements are taken every time step, but that control is applied only every $3^{\text {rd }}$ step. The WF estimate error trends smaller with each step, even when controls are not applied.

- The performance of this single case is well under the WFE error tree "Model Only" total of $5.3 \mathrm{um}$, as discussed in Section 7 and summarized in Figure 11.
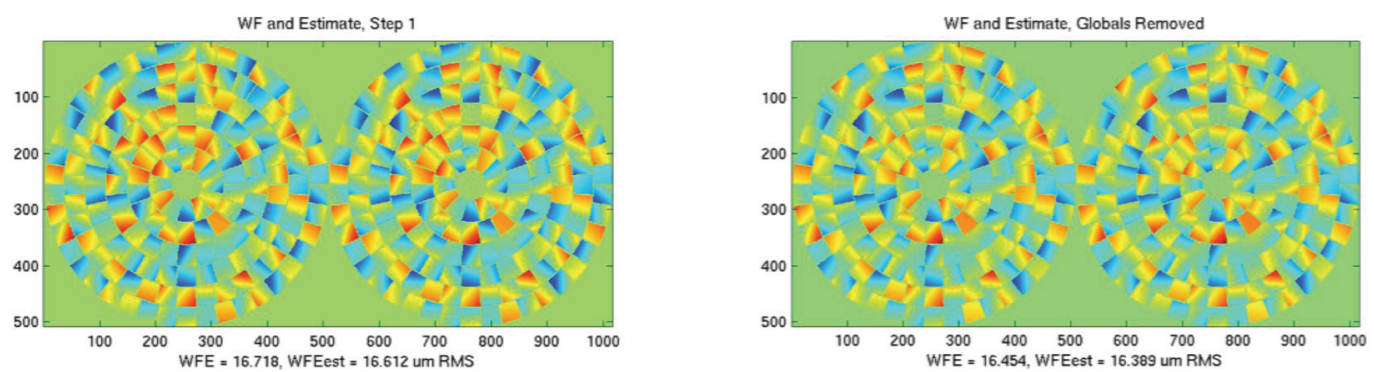

Figure 15. Initial condition recovery simulation example, time step 1, showing initial WF and initial estimated WF, with all modes included (left plot) and with unobservable global modes removed (right plot).
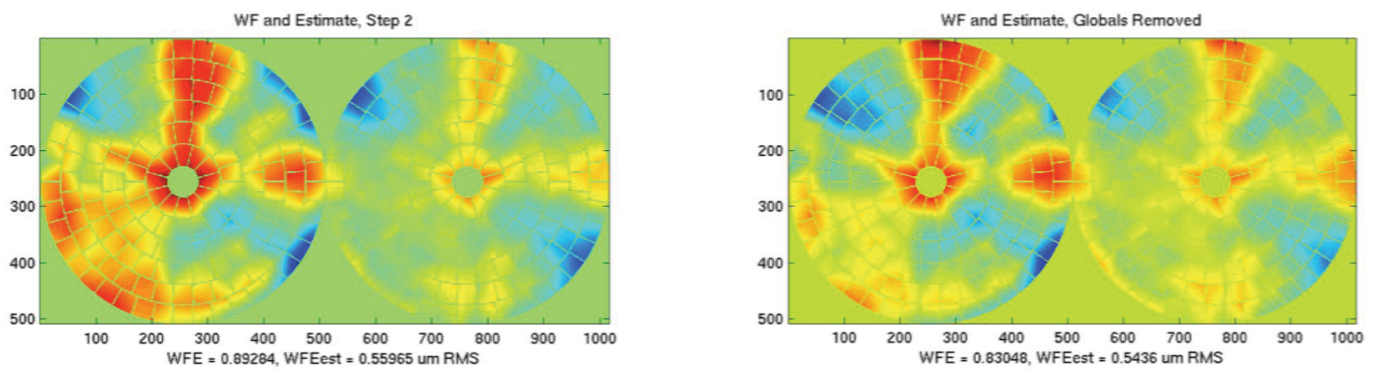

Figure 16. Initial condition recovery simulation example, time step 2, showing WF and estimated WF after the first control step. 

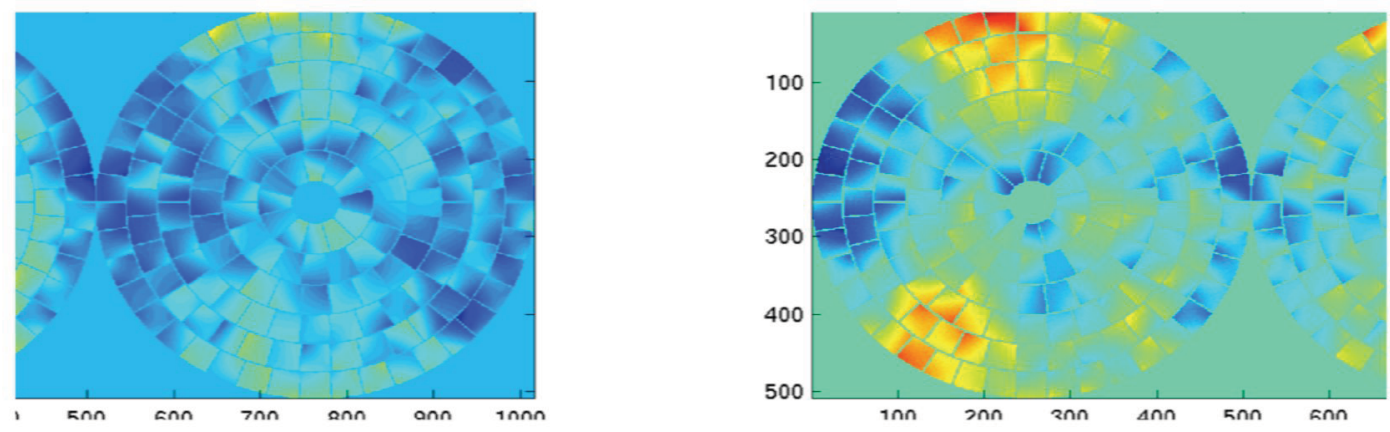

Figure 17. Initial condition recovery simulation example, time step 22. Errors are small, and dominated by measurement-noise driven estimation error, since process noise and actuation error are small in this example.

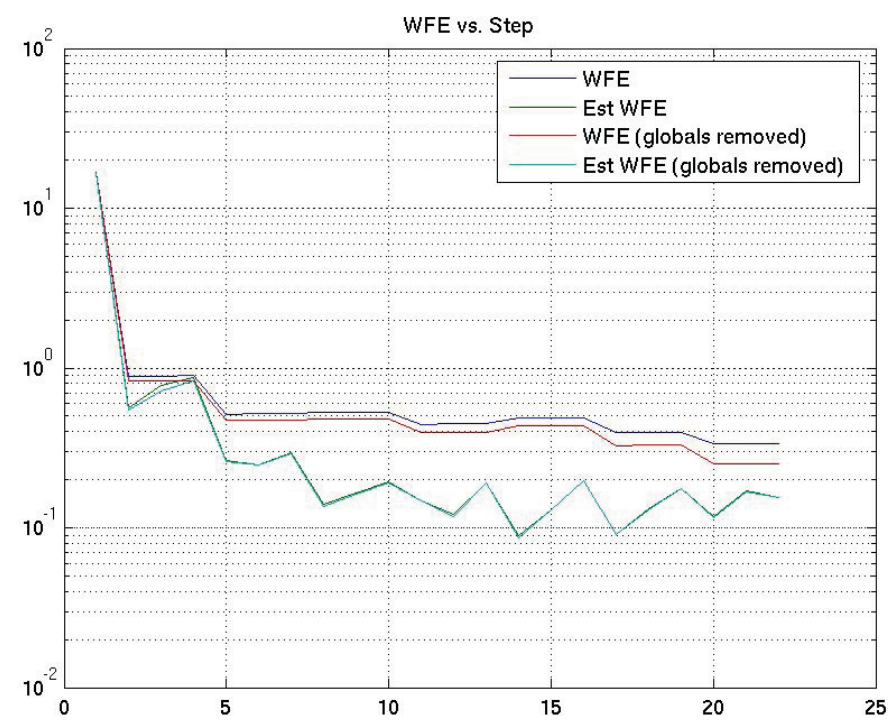

Figure 18. Wavefront error vs. time step, showing actual WFE (blue trace), WFE with global modes removed (red trace) and the Estimate Error (green trace).

\subsection{WF Maintenance Control examples with fixed gain OSE.}

This section repeats the conditions of the example of Section 7.1, for 3 different fixed gain estimators. The first case uses $K_{0}$, the first-step OSE gains, computed based on a large $X_{0}$ consistent with the simulated disturbance. The results, presented in Figure 19, show that control for the first step matches that of the full OSE - no surprise there. As time progresses, though, the fixed-gain OSE performance does not improve like that of the full OSE. The fixed-gain version does not adjust the gains as the state errors decrease, and the WF errors at the end of the 22 step run are about 1.6 um: twice those of the full Kalman filter OSE. Nonetheless, fixed-gain performance using $K_{0}$ remains substantially better than the target value of $5.3 \mathrm{um}$.

The second fixed-gain case uses gains computed using the OSE after 12 time steps, when the state errors have dropped considerably. These gains favor the prior state estimate more, and are not as responsive to the large initial condition error. The result, as shown in Figure 20, is a slower recovery from the initial error, achieving only about 3.5 um error by the 18th time step; it is likely that the ultimate performance will be substantially better - but the slow time response is not desirable. 


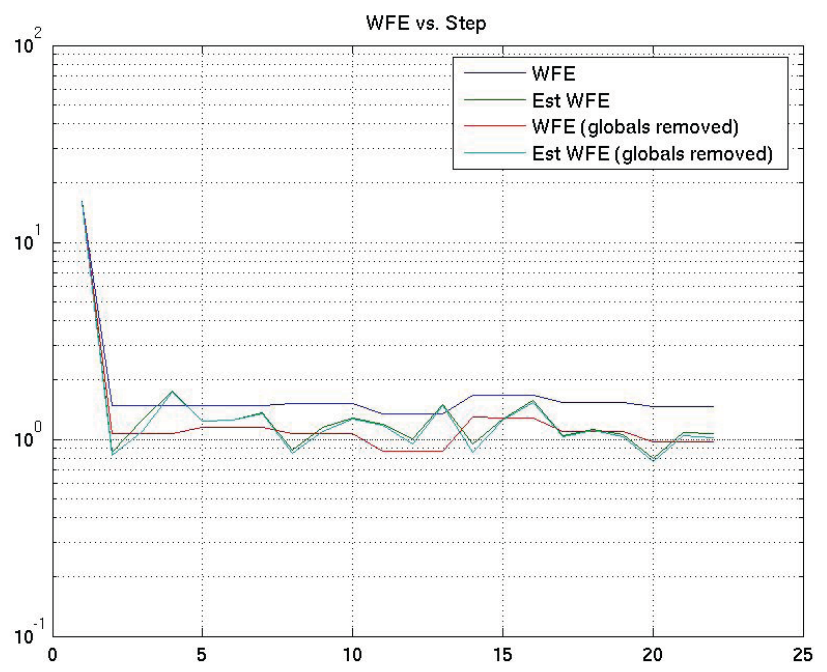

Figure 19. Fixed-gain time series example, with $K_{0}$ based on large initial-condition errors.

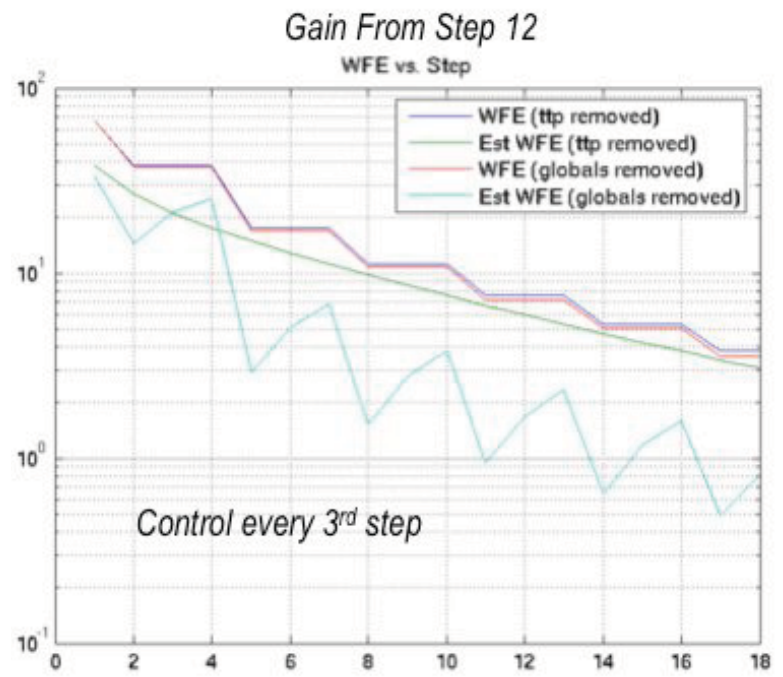

Figure 20. Fixed-gain time series example, with $K_{I 2}$ computed for the $12^{\text {th }}$ time step, with smaller state errors. Much slower convergence is observed.

The third case utilizes a "gain scheduling" approach. When errors are large the $K_{0}$ gains are used. After 12 steps, when the errors have decreased substantially, the gains are switched to the $K_{12}$ gains. The result is control performance that compares very favorably to the full, time-varying gain OSE: achieving under 1 um performance by the $18^{\text {th }}$ step.

The gain scheduling approach provides a compromise between good WF performance and a desire to keep real-time computational burdens low - but current performance projections do not show that it is needed to meet the error tree performance allocations. For this reason, we consider the fixed-gain approach, using the $K_{0}$ gains, to be the baseline.

\subsection{Gravity sag disturbances: WF Initialization.}

As discussed in Section 3, the largest disturbance for CCAT WF Control is the structural deformation experienced as the telescope elevation angle varies. WF control performance under this condition is explored in this subsection.

WF Sensing used to measure gravity sag. The first WF control example provides a simple simulation of the use of WF sensing for the initial commissioning of the telescope, with the performance history summarized on Figure 22. It begins with gravity sag errors applied to the telescope, but with no feed-forward control. The resulting large WFE is illustrated in Figure 23. The gravity disturbance has large global and non-global components; the latter are visible to the IDS and 
are corrected in the first few steps, as seen in Figure 24. The estimate at that time is very small, as the large global WF modes which remain uncorrected are not observable by the IDS.

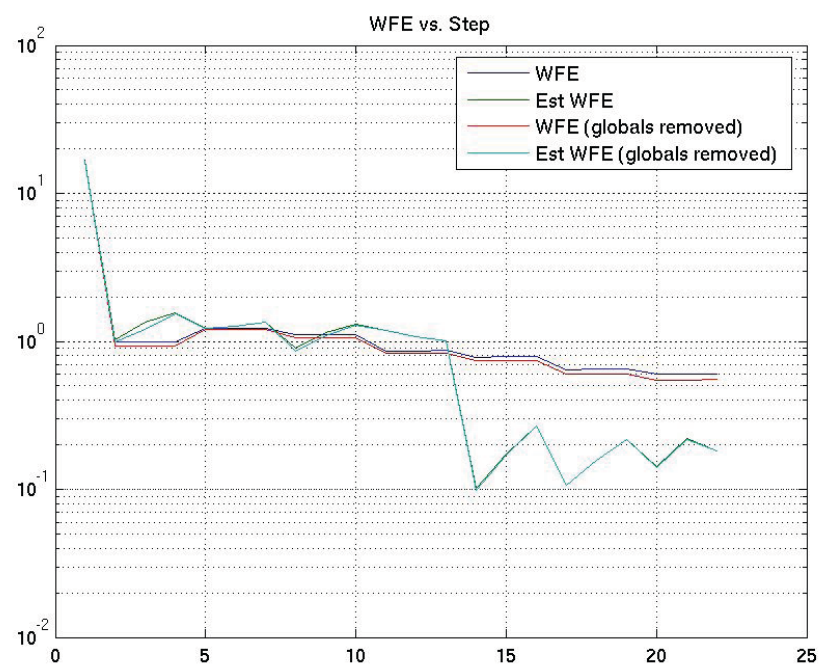

Figure 21. Gain-scheduled control. Fixed gain is switched at time step 12. Initial response using gain $K_{0}$ is rapid, but saturates at a relatively high value. Performance improves after the large initial error is corrected through use of gain $K_{12}$.

WF Sensing is conducted at time step 6, suddenly making it possible to see the global mode errors, as shown in Figure 25. The control that occurs after the WFS update, at step 7, effectively removes both the global and local mode errors (Figure 26). The WFS update is used following Eq. 32.

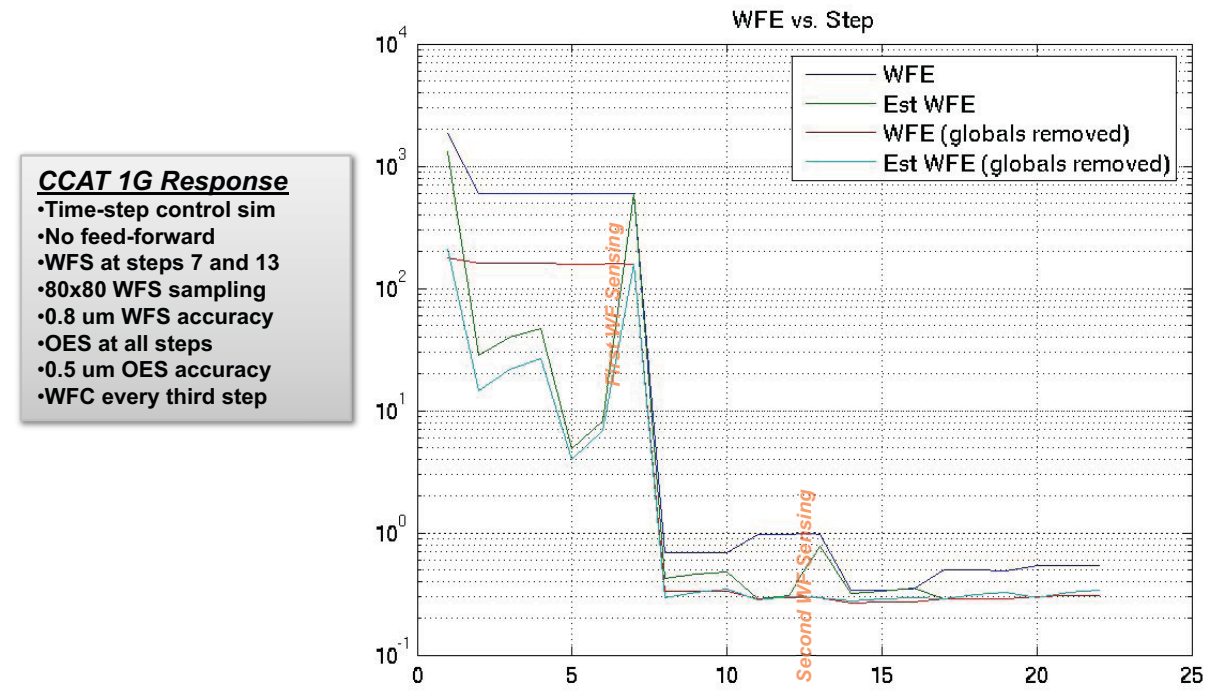

Figure 22. WFE vs. time step, for $1 \mathrm{G}$ sag due to change in elevation angle from zenith pointing to $45^{\circ}$ off zenith. No feed-forward is used in this case. WF Sensing is conducted at steps 6 and 13. This case uses the full Kalman variable-gain OSE. 


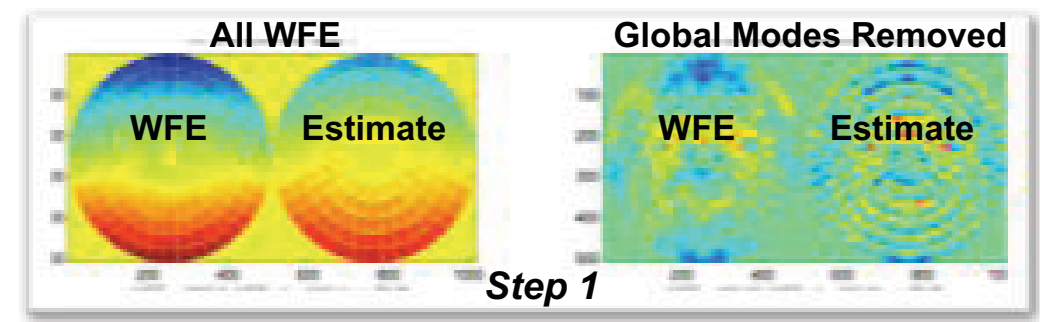

-Initial WFE due to gravity sag

-Initial WF estimate does not observe global WFE

Figure 23. WFE and estimated WFE, both with and without global modes, at initial time step, for $1 \mathrm{G}$ example.

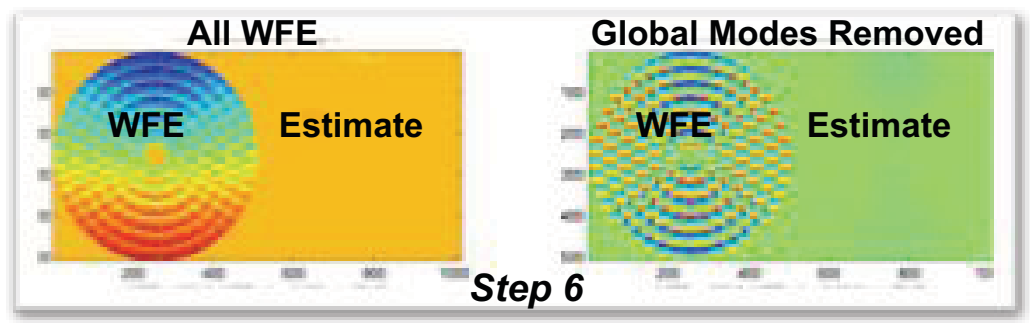

-WFE after OES control only

-Estimates do not see global mode WFE

-OES control only does not correct global mode WFE

Figure 24. WFE and estimated WFE, both with and without global modes, at time step 6, for 1G example. The IDS measurements have enabled the controller to correct the PM modes, driving the estimated WFE towards zero; the unobservable global mode WFE remains.

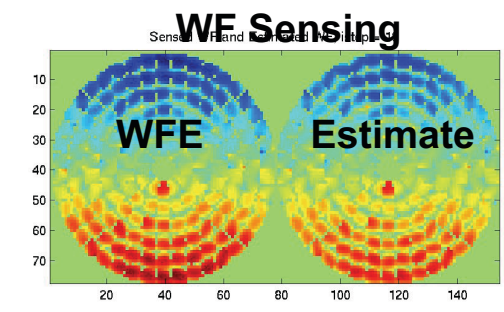

Figure 25. The result of WF sensing at time step 6. The actual WFE and the WFE estimate obtained by feeding the WF measurement through the OSE agree very closely. WFS error was 0.8 um 1-sigma per pixel, with 80 by-80 samples in the estimate.

Performance at the conclusion of the $22^{\text {nd }}$ time step in this example was well under 1 um, growing slowly, driven by the process and IDS sensing noise, towards the sensing noise floor of about $1 \mathrm{um}$ (Figure 32). This performance with the full OSE is better than the targets set by the error tree. 


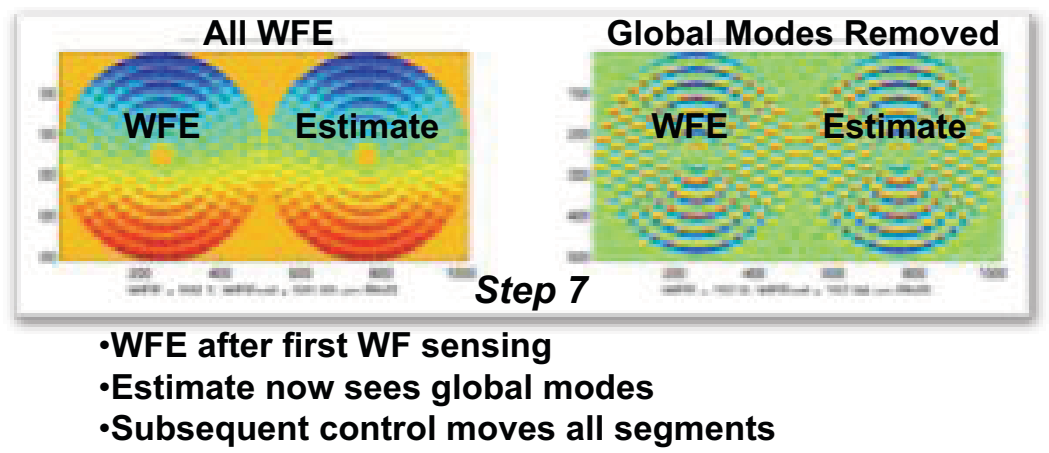

Figure 26. WFE and estimated WFE, both with and without global modes, at time step 7, for 1G example. The first IDS measurement after WFS-based control shows excellent agreement between the estimate and actual WFE. Control for this step will include global modes.

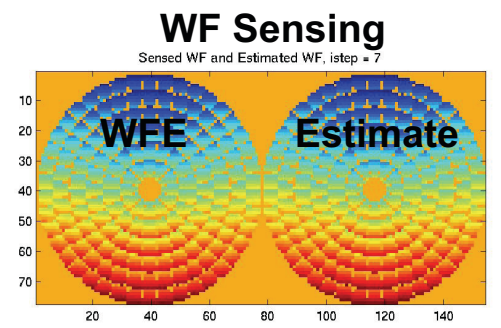

Figure 27. The result of WF sensing at time step 13. Again, the actual WFE and the WFE estimate obtained by feeding the WF measurement through the OSE agree very closely. WFS error was 0.8 um 1-sigma per pixel, with 80 -by-80 samples in the estimate. WFS error per segment averaged $0.16 \mathrm{um}$.

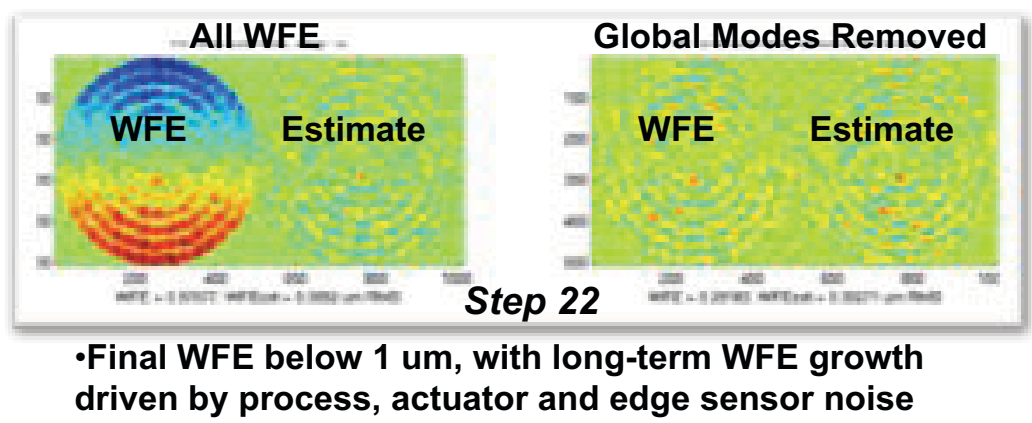

Figure 28. WFE and estimated WFE, both with and without global modes, at time step 22 .

Fixed-gain example: using WF sensing for measurement of gravity sag. The same example was run using the fixedgain OSE, with gains $K_{12}$, with the results seen in Figure 29. Here the excellent WFS performance is preserved by using the gains appropriate to small state error. 


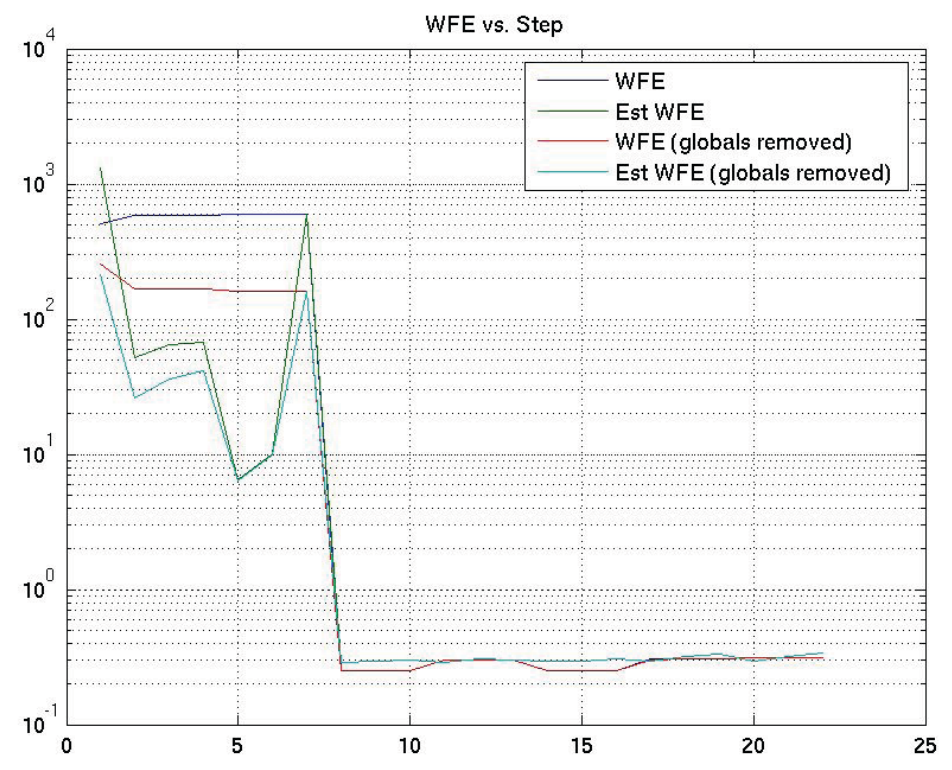

Figure 29. WFE vs. time step, for $1 \mathrm{G}$ sag due to change in elevation angle from zenith pointing to $45^{\circ}$ off zenith. No feed-forward is used in this case. WF Sensing is conducted at steps 6 and 13. This case uses fixed-gain OSE, with $K_{12}$ gains.

Once again, a fixed-gain controller appears to handily meet the error performance targets.

Feed-Forward control example. There will be few opportunities to conduct WF sensing with CCAT, due to the sparse availability of calibrations sources such as Mars and Uranus. Compensation of WFE due to $1 \mathrm{G}$ sag will therefore be by feed-forward control based on a Look-Up Table, as discussed above. This situation is simulated in Figure 30 for the case of a fixed $K_{0}$ gain control, with $500 \mathrm{~nm}$ IDS sensing error RMS.

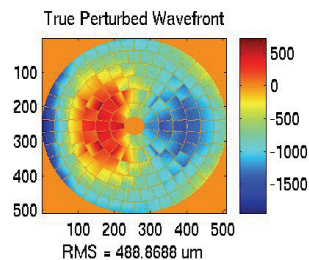

RMS $=488.8688 \mathrm{um}$
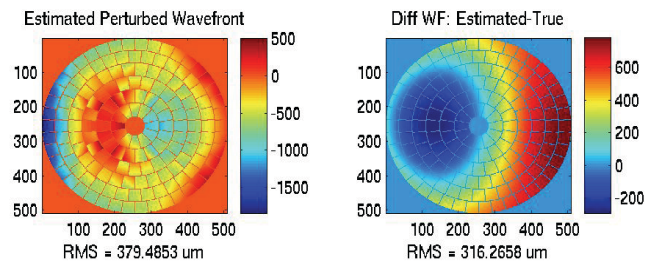
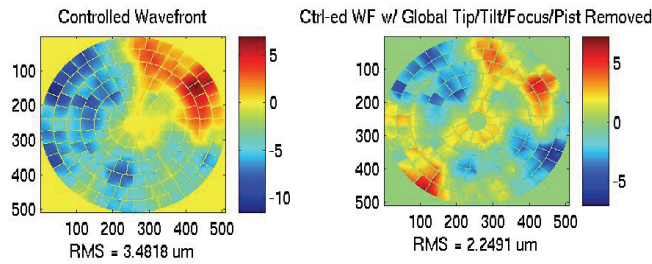

Figure 30. WFE before and after control, $1 \mathrm{G}$ sag deformation case. The difference between the true and estimated WF is due to global motion of the PM and SM; the control uses LUT feed-forward to move the SM to compensate, and the IDS signals to phase the segments.

Thermal "cupping" example. An approximate analysis of "cupping" effects has been begun. Cupping refers to the curvature change experienced by the segment structures in response to thermal gradients or $\triangle \mathrm{CTE}$ effects. Analysis by CCAT system engineering identified a likely cupping effect from thermal gradients of 1 um peak-to-valley, affecting the optical surface, and also changing the orientation of the IDS sensor head mounting pads. WFE is affected directly, because the reflecting surface bends; and indirectly, because the sensor geometry changes lead to spurious error signals.

Cupping effects, both the reflecting surface deformation and the change in sensor geometry, have been modeled and we have begun to explore the performance impacts. As an example, Figure 31 repeats the simulation of Figure 30, adding a uniform 1 um cupping to each segment surface and to all IDS sensors. No attempt was made to measure or control the additional error. Standard LUT feed-forward control of the SM was applied, and the IDS was used with fixed $K_{0}$ gains and $500 \mathrm{~nm}$ IDS noise RMS. The results, seen in Figure 31, are slightly worse than those of Figure 30, but still within the target WFE of $5.3 \mathrm{um}$. 

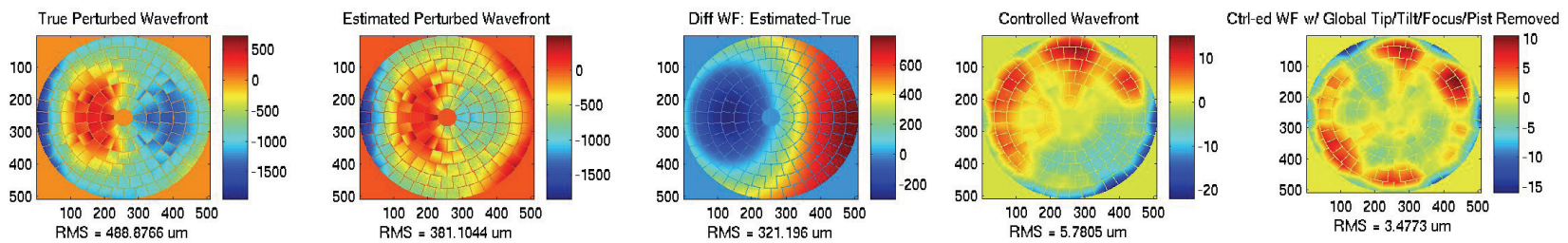

Figure 31. WFE before and after control, $1 \mathrm{G}$ sag deformation case, including segment cupping, for comparison with Figure 30.

\subsection{Sensing noise.}

WF Maintenance control performance is affected by noise in the IDS sensors. This is explored using Monte Carlo simulation for the case of the $K_{0}$ fixed-gain controller in Figure 32. Fifty simulations were run for each datapoint, with results bounded as shown.

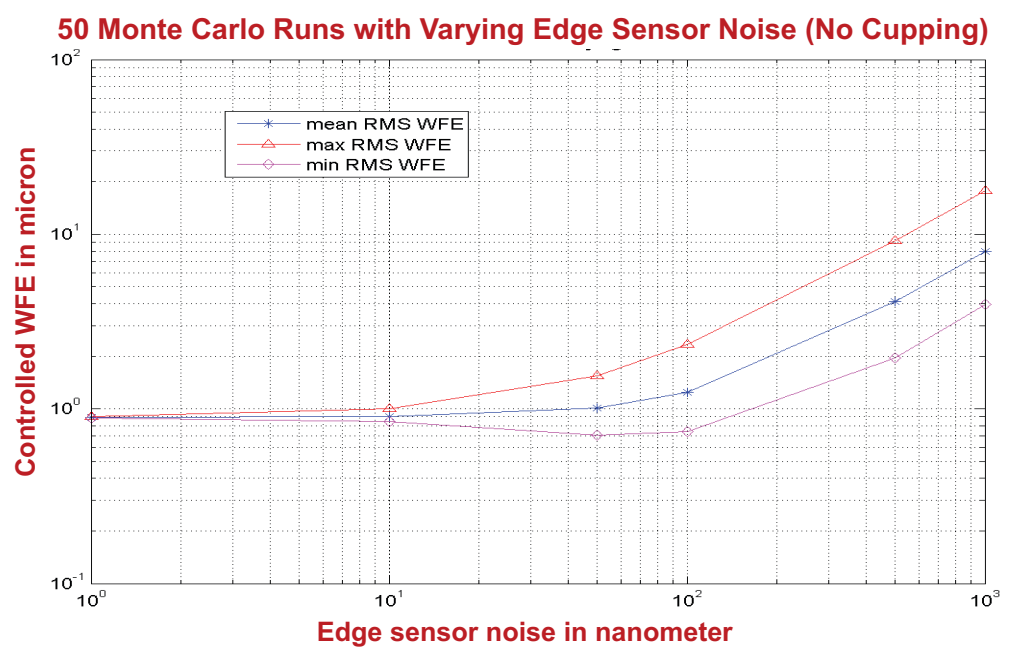

Figure 32. Monte Carlo analysis, varying IDS sensor noise from 1 to 1,000 nm RMS. Fixed-gain OSE, using $K_{0}$ gains; no thermal gradient cupping. Noise floor of about $1 \mathrm{um}$ is set by residuals of initial state errors.

The same analysis was run for a set of cases including uniform cupping of 1 um peak-to-valley, as shown in Figure 33. The difference here is in the noise floor - the asymptote at the low end of the IDS noise curve. The cupping is seen to raise the floor from $1 \mathrm{um}$ for the no-cupping case, to about 3 um with cupping present. Performance when IDS noise is $100 \mathrm{~nm}$ is not much worse. The WFE remains below the $5.3 \mathrm{um}$ target for IDS noise values up to $600 \mathrm{~nm}$ RMS.

\section{Conclusion}

CCAT Wavefront Control provides continuous wavefront maintenance control using Imaging Displacement Sensors to measure segment-to-segment motions. It uses occasional Wavefront Sensing to initialize the telescope and recalibrate the maintenance control. The control system uses a full state feedback approach, with an Optical State Estimator to estimate the optical state and predict wavefront error, and then uses this estimate in a controller designed to minimize wavefront error. This design permits effective control, not only of errors in the directly-controlled degrees of freedom, but also of the optical effects of errors in uncontrolled degrees of freedom.

Preliminary results indicate that control performance will meet CCAT requirements, even with a conservative implementation approach: fixed-gain estimator rather than a full Kalman filter; no attempt to control segment thermal deformations; feed-forward control for correction of gravity sag effects.

Much work remains. Most important is to complete the design of a Zernike WF sensor, and to test it on the sky. Further work will refine and update the models of CCAT, to add dynamics, to incorporate design changes, to assess the effects of model error, to provide a more thorough performance assessment, to more fully explore the range of CCAT operating conditions and to support more detailed design trade studies. 
This research was carried out at the Jet Propulsion Laboratory, California Institute of Technology, under a contract with the National Aeronautics and Space Administration.

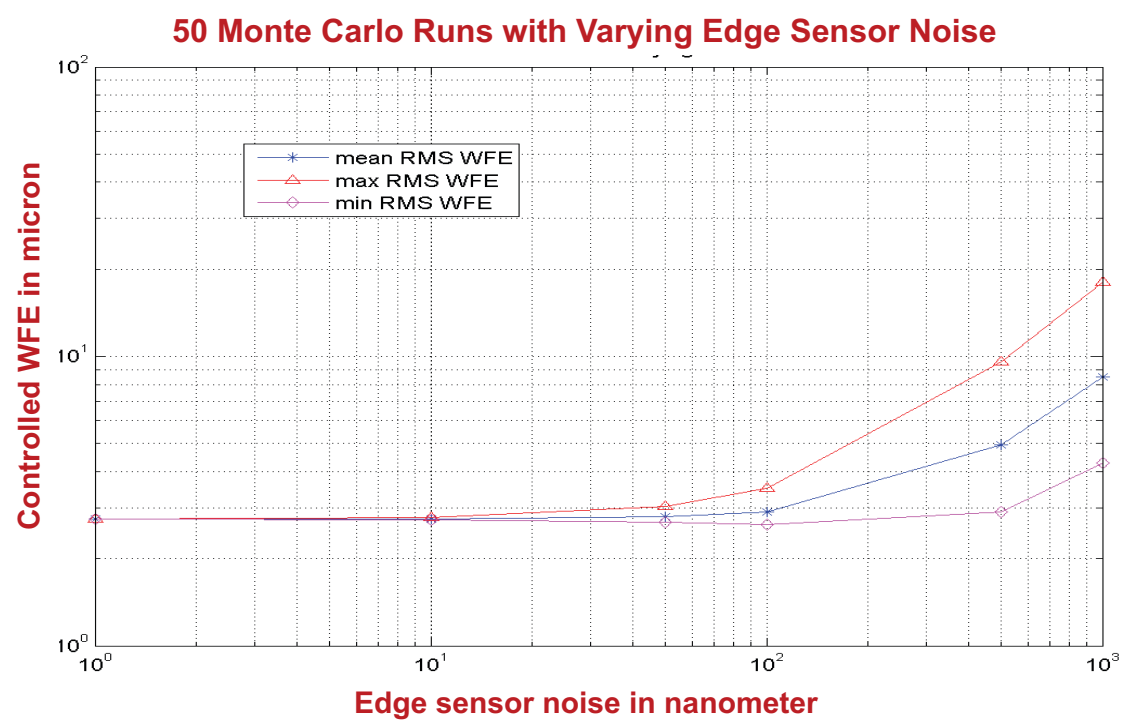

Figure 33. Monte Carlo analysis, varying IDS sensor noise from 1 to 1,000 nm RMS. The higher noise floor of about 3 um is set by residuals of initial state errors and cupping effects.

\section{REFERENCES}

[1] Cornell Caltech Atacama Telescope (CCAT) Feasibility and Concept Design Study, Final Report (2006).

[2] D. Redding, J.Z. Lou, A. Kissil, J.K. Wallace, E. Serabyn, C.M. Bradford, S. Padin and D. Woody, CCAT Wavefront Control Interim Report, JPL Document (2011).

[3] S. Padin, CCAT Optical Design for $1^{\circ} \mathrm{FOV}$, CCAT Tech Memo \#47, October, 2009

[4] MACOS Manual, JPL Document (1999).

[5] D. Woody, Conceptual Design for the CCAT Primary and Tipping Structure, Ver. 2.1, CCAT Tech Memo \#41, August, 2009.

[6] CCAT Telescope Project Book, http://wiki.astro.cornell.edu/twiki/pub/CCAT/Home/TelescopeProjectBook.pdf (2011).

[7] S. Padin and D. Woody, "CCAT Error Budget," http://wiki.astro.cornell.edu/twiki/ (2011).

[8] D. Redding and W. Breckenridge, "Linearized Ray-Trace Analysis," Selected SPIE Papers on CD ROM, Volume 2, Lens Design, D. O'Shea, ed. (1998).

[9] D. Woody, D. Redding, S. Padin and B. Tong, "An imaging displacement sensor with nanometer precision and high stability," Caltech report (2011).

[10] E. Serabyn, T. Phillips and C. Masson, "Surface Figure Measurements of Radio Telescopes with a Shearing Interferometer," Applied Physics, Vol. 30, No. 10 (1991).

[11] J.Z. Lou, A. Kissil, D. Redding, C.M. Bradford, S.Padin and D. Woody, "Modeling a Large Submillimeter-Wave Observatory," SPIE (2010).

[12]A. Abramovici, "Electro-Optic Measurement of CCAT Segment-Segment and Primary-Secondary Relative Motions," JPL Internal Document (2009).

[13] D. MacDonald, D. Woody, C. Bradford, R. Chamberlin, M. Dragovan, S. Radford, T. Sebring, J. Zmuidzinias, and P. Goldsmith, "Cornell Caltech Atacama Telescope Primary Mirror Surface Sensing and Controllability," SPIE 7012, (2008).

[14] A. Bryson and Y. Ho, Applied Optimal Control, Hemisphere Publishing (1975).

[15] D. Redding, B. Breckenridge, Ken Lau, G. Sevaston, M. Levine and S. Shaklan, "Segmented Mirror Figure Control for a Space-Based Far-IR Astronomical Telescope," SPIE 1489 (1991). 